\title{
Asymptotic behavior of critical, irreducible multi-type continuous state and continuous time branching processes with immigration
}

\author{
MÁtyás BARCZY*,», Gyula PAP** \\ * Faculty of Informatics, University of Debrecen, Pf. 12, H-4010 Debrecen, Hungary. \\ ** Bolyai Institute, University of Szeged, Aradi vértanúk tere 1, H-6720 Szeged, Hungary. \\ e-mails: barczy.matyas@inf.unideb.hu (M. Barczy), papgy@math.u-szeged.hu (G. Pap). \\ $\diamond$ Corresponding author.
}

\|\|

\begin{abstract}
Under natural assumptions, a Feller type diffusion approximation is derived for critical, irreducible multi-type continuous state and continuous time branching processes with immigration. Namely, it is proved that a sequence of appropriately scaled random step functions formed from a critical, irreducible multi-type continuous state and continuous time branching process with immigration converges weakly towards a squared Bessel process supported by a ray determined by the Perron vector of a matrix related to the branching mechanism of the branching process in question.
\end{abstract}

\section{Introduction}

The study of the limit behaviour of critical multi-type branching processes has a long tradition and history. Most of the literature is devoted to so-called conditioned limit theorems for branching processes without immigration.

For a certain class of critical positively regular discrete time branching processes without immigration $\left(Z_{n}\right)_{n \geqslant 1}$ with a finite or infinite number of types, Mullikin [24, Theorem 9] characterized the conditional limiting distribution of $n^{-1} Z_{n}$ given that $Z_{n} \neq 0$ as $n \rightarrow \infty$.

For critical discrete time branching processes with immigration $\left(X_{n}\right)_{n \geqslant 1}$, under second order moment assumptions, Wei and Winnicki [28, Theorem 2.1] proved weak convergence of a sequence of step processes $\left(n^{-1} X_{\lfloor n t\rfloor}\right)_{t \geqslant 0}, n \geqslant 1$, as $n \rightarrow \infty$, characterizing the limit process as a squared Bessel process.

2010 Mathematics Subject Classifications: 60J80,60F17.

Key words and phrases: critical irreducible multi-type branching processes with immigration, squared Bessel processes.

The research was realized in the frames of TÁMOP 4.2.4. A/2-11-1-2012-0001 „National Excellence Program - Elaborating and operating an inland student and researcher personal support system". The project was subsidized by the European Union and co-financed by the European Social Fund. 
Ren et al. 25] investigated conditional limit theorems for critical continuous-state and continuous time branching processes $\left(X_{t}\right)_{t \geqslant 0}$ with branching mechanism $\lambda^{1+\alpha} L(1 / \lambda)$, but without immigration, where $\alpha \in[0,1]$ and $L$ is slowly varying at $\infty$. They proved that if $\alpha \in(0,1]$, then there are norming constants $Q_{t} \rightarrow 0$ (as $t \uparrow \infty$ ) such that for every $x>0$, $P_{x}\left(Q_{t} X_{t} \in \cdot \mid X_{t}>0\right)$ converges weakly to a non-degenerate limit as $t \rightarrow \infty$. As a continuation of these investigations, Ren et al. [26] studied conditional limit theorems for some critical superprocesses conditioned on non-extinction.

Recently, Iyer et al. [18, Section 7] investigated limit theorems for critical continuous time and continuous state branching processes (without immigration) that become extinct almost surely. First, they obtained a conditional limit theorem for fixed initial population size, and next, they studied non-conditioned scaling limits with initial population size scaled to obtain non-degenerate Lévy process limits.

For a single-type (one-dimensional) critical continuous state and continuous time branching process with immigration (CBI process) $X$, under second order moment assumptions, Huang et al. [13, Theorem 2.3] characterized the limiting distribution of $\left(n^{-1} X_{\lfloor n t\rfloor}\right)_{t \geqslant 0}$ as $n \rightarrow \infty$. Our Theorem 4.1 is a generalization of this result to the multi-type case (under fourth order moment assumptions), see Remark 4.5, and it may serve as a key tool for studying asymptotic behaviour of conditional least squares estimators of some parameters of processes in question.

The paper is organized as follows. In Section 2, for completeness and better readability, we recall from Barczy et al. [4] some notions and statements for multi-type CBI processes such as the form of their infinitesimal generator, and a formula for their first moment. In Section 3 , we introduce irreducible CBI processes and we give a classification, namely we define subcritical, critical and supercritical irreducible CBI processes, see Definitions 3.1] and 3.2, respectively.

In Section 4, it is proved that the sequence $\left(n^{-1} \boldsymbol{X}_{\lfloor n t\rfloor}\right)_{t \geqslant 0}, n \geqslant 1$, of scaled random step functions formed from a critical, irreducible multi-type CBI process $\boldsymbol{X}$ converges weakly towards a squared Bessel process supported by a ray determined by the Perron vector of a matrix related to the branching mechanism of $\boldsymbol{X}$, see Theorem 4.1. The limit process is characterized as a pathwise unique strong solution of a stochastic differential equation (SDE). In Remark 4.5, we specialize Theorem 4.1 to dimension 1 noting that the limit process is a single-type CBI diffusion process. In Remark 4.6 we point out that in case of $d \geqslant 2$, the limit process is not a $d$-type CBI process any longer, contrary to dimension 1 . We also formulate a consequence of Theorem 4.1 deriving a limit distribution for the relative frequencies of distinct types of individuals, see Corollary 4.7. For different models, one can find similar results in Jagers [20, Corollary 1] and in Yakovlev and Yanev [29, Theorem 2]. Section 5 is devoted to give examples for multi-type CBI processes so that the drift and diffusion coefficients of the SDE characterizing the limit process in Theorem 4.1 are calculated explicitly. In Section 6 we prove Theorem 4.1. First, we prove weak convergence of a sequence of martingale differences $\left(\boldsymbol{M}_{n}\right)_{n \geqslant 1}$ constructed from an irreducible and critical multi-type CBI process $\boldsymbol{X}$. Namely, $\boldsymbol{M}_{n}$ is the difference of $\boldsymbol{X}_{n}$ and the conditional expectation of $\boldsymbol{X}_{n}$ with respect to $\boldsymbol{X}_{n-1}$. The proof is based on a result due to Ispány and Pap [16, Corollary 2.2] (see also Theorem 
C.1), which is about convergence of random step processes towards a diffusion process. Using weak convergence of $\left(\boldsymbol{M}_{n}\right)_{n \geqslant 1}$, an application of a version of the continuous mapping theorem (see Lemma D.1) yields weak convergence of $\left(n^{-1} \boldsymbol{X}_{\lfloor n t\rfloor}\right)_{t \geqslant 0}$ as $n \rightarrow \infty$. Comparing our proof of technique with that of Huang et al. [13, Theorem 2.3] (where the dimension is 1), they are completely different. Huang et al. [13] start with a SDE with jumps for the process $X$, and, applying Doob's inequality, tightness of the scaled processes $\left(n^{-1} X_{n t}\right)_{t \geqslant 0}, n \geqslant 1$, is shown. Then, by Skorokhod's theorem and a semimartingale representation theorem, they prove weak convergence of $\left(n^{-1} X_{n t}\right)_{t \geqslant 0}$ as $n \rightarrow \infty$ characterizing the limit distribution as well. Finally, an application of the continuous mapping theorem yields weak convergence of $\left(n^{-1} X_{\lfloor n t\rfloor}\right)_{t \geqslant 0}$ as $n \rightarrow \infty$ with the same limit distribution. Comparing our technique of proof with that of Wei and Winnicki [28, Theorem 2.1], one can realize that they are completely different as well. They calculated the infinitesimal generator of step processes $\left(n^{-1} X_{\lfloor n t\rfloor}\right)_{t \geqslant 0}$, $n \geqslant 1$, and examined its limit behaviour as $n \rightarrow \infty$ resulting the infinitesimal generator of the limit squared Bessel process. In a companion paper Barczy and Pap [6, Corollary 3.5 and Remark 3.6] we investigate convergence properties of the sequence of infinitesimal generators of $\left(n^{-1} \boldsymbol{X}_{\lfloor n t\rfloor}\right)_{t \geqslant 0}, \quad n \geqslant 1$. It is an open question whether our main Theorem 4.1 might be proved by the help of infinitesimal generators. Further, we note that, to the best knowledge of the authors, it is not known, whether the sequence of scaled processes $\left(n^{-1} \boldsymbol{X}_{n t}\right)_{t \geqslant 0}, n \geqslant 1$, is weakly convergent for an irreducible and critical $d$-type CBI process $\boldsymbol{X}$ with $d \geqslant 2$.

In Appendix $\mathrm{A}$ we present some Frobenius-Perron type results for irreducible matrices having non-negative off-diagonal entries. Appendix B is devoted to study asymptotic behaviour of moments of irreducible and critical multi-type CBI processes. First, we describe the asymptotic behaviour of the first moment of irreducible multi-type CBI processes, see Proposition B.1. The classification given in Definition 3.2 is based on this description. In case of an irreducible and critical multi-type CBI process $\boldsymbol{X}$, we derive some moment estimations for the process and the corresponding sequence of martingale differences $\left(\boldsymbol{M}_{n}\right)_{n \geqslant 1}$, see Lemmas B.2 and B.3, respectively. In Appendix C, we recall a result about convergence of random step processes towards a diffusion process due to Ispány and Pap [16, Corollary 2.2]. In Appendix D we present a version of the continuous mapping theorem.

\section{Multi-type CBI processes}

Let $\mathbb{Z}_{+}, \mathbb{N}, \mathbb{R}, \mathbb{R}_{+}$and $\mathbb{R}_{++}$denote the set of non-negative integers, positive integers, real numbers, non-negative real numbers and positive real numbers, respectively. For $x, y \in \mathbb{R}$, we will use the notations $x \wedge y:=\min \{x, y\}$ and $x^{+}:=\max \{0, x\}$. By $\|\boldsymbol{x}\|$ and $\|\boldsymbol{A}\|$, we denote the Euclidean norm of a vector $\boldsymbol{x} \in \mathbb{R}^{d}$ and the induced matrix norm of a matrix $\boldsymbol{A} \in \mathbb{R}^{d \times d}$, respectively. The natural basis in $\mathbb{R}^{d}$ and the Borel $\sigma$-algebras on $\mathbb{R}^{d}$ and on $\mathbb{R}_{+}^{d}$ will be denoted by $\boldsymbol{e}_{1}, \ldots, \boldsymbol{e}_{d}$, and by $\mathcal{B}\left(\mathbb{R}^{d}\right)$ and $\mathcal{B}\left(\mathbb{R}_{+}^{d}\right)$, respectively. The $d$-dimensional unit matrix is denoted by $\boldsymbol{I}_{d}$. For $\boldsymbol{x}=\left(x_{i}\right)_{i \in\{1, \ldots, d\}} \in \mathbb{R}^{d}$ and $\boldsymbol{y}=\left(y_{i}\right)_{i \in\{1, \ldots, d\}} \in \mathbb{R}^{d}$, we will use the notation $\boldsymbol{x} \leqslant \boldsymbol{y}$ indicating that $x_{i} \leqslant y_{i}$ for all $i \in\{1, \ldots, d\}$. By $C_{\mathrm{c}}^{2}\left(\mathbb{R}_{+}^{d}, \mathbb{R}\right)$ we 
denote the set of twice continuously differentiable real-valued functions on $\mathbb{R}_{+}^{d}$ with compact support. Throughout this paper, we make the conventions $\int_{a}^{b}:=\int_{(a, b]}$ and $\int_{a}^{\infty}:=\int_{(a, \infty)}$ for any $a, b \in \mathbb{R}$ with $a<b$.

2.1 Definition. A matrix $\boldsymbol{A}=\left(a_{i, j}\right)_{i, j \in\{1, \ldots, d\}} \in \mathbb{R}^{d \times d}$ is called essentially non-negative if $a_{i, j} \in \mathbb{R}_{+}$whenever $i, j \in\{1, \ldots, d\}$ with $i \neq j$, i.e., if $\boldsymbol{A}$ has non-negative off-diagonal entries. The set of essentially non-negative $d \times d$ matrices will be denoted by $\mathbb{R}_{(+)}^{d \times d}$.

2.2 Definition. A tuple $(d, \boldsymbol{c}, \boldsymbol{\beta}, \boldsymbol{B}, \nu, \boldsymbol{\mu})$ is called a set of admissible parameters if

(i) $d \in \mathbb{N}$,

(ii) $\boldsymbol{c}=\left(c_{i}\right)_{i \in\{1, \ldots, d\}} \in \mathbb{R}_{+}^{d}$,

(iii) $\boldsymbol{\beta}=\left(\beta_{i}\right)_{i \in\{1, \ldots, d\}} \in \mathbb{R}_{+}^{d}$,

(iv) $\boldsymbol{B}=\left(b_{i, j}\right)_{i, j \in\{1, \ldots, d\}} \in \mathbb{R}_{(+)}^{d \times d}$,

(v) $\nu$ is a Borel measure on $U_{d}:=\mathbb{R}_{+}^{d} \backslash\{\mathbf{0}\}$ satisfying $\int_{U_{d}}(1 \wedge\|\boldsymbol{z}\|) \nu(\mathrm{d} \boldsymbol{z})<\infty$,

(vi) $\boldsymbol{\mu}=\left(\mu_{1}, \ldots, \mu_{d}\right)$, where, for each $i \in\{1, \ldots, d\}, \mu_{i}$ is a Borel measure on $U_{d}$ satisfying

$$
\int_{U_{d}}\left[\|\boldsymbol{z}\| \wedge\|\boldsymbol{z}\|^{2}+\sum_{j \in\{1, \ldots, d\} \backslash\{i\}} z_{j}\right] \mu_{i}(\mathrm{~d} \boldsymbol{z})<\infty .
$$

2.3 Remark. Our Definition 2.2 of the set of admissible parameters is a special case of Definition 2.6 in Duffie et al. 9], which is suitable for all affine processes, see Barczy et al. 4, Remark 2.3]. Further, for all $i \in\{1, \ldots, d\}$, condition (2.1) is equivalent to

$$
\int_{U_{d}}\left[\left(1 \wedge z_{i}\right)^{2}+\sum_{j \in\{1, \ldots, d\} \backslash\{i\}}\left(1 \wedge z_{j}\right)\right] \mu_{i}(\mathrm{~d} \boldsymbol{z})<\infty \quad \text { and } \quad \int_{U_{d}}\|\boldsymbol{z}\| \mathbb{1}_{\{\|\boldsymbol{z}\| \geqslant 1\}} \mu_{i}(\mathrm{~d} \boldsymbol{z})<\infty
$$

see Barczy et al. [4, Remark 2.3].

2.4 Theorem. Let $(d, \boldsymbol{c}, \boldsymbol{\beta}, \boldsymbol{B}, \nu, \boldsymbol{\mu})$ be a set of admissible parameters. Then there exists a unique conservative transition semigroup $\left(P_{t}\right)_{t \in \mathbb{R}_{+}}$acting on the Banach space (endowed with the supremum norm) of real-valued bounded Borel-measurable functions on the state space $\mathbb{R}_{+}^{d}$ such that its infinitesimal generator is

$$
\begin{aligned}
(\mathcal{A} f)(\boldsymbol{x})= & \sum_{i=1}^{d} c_{i} x_{i} f_{i, i}^{\prime \prime}(\boldsymbol{x})+\left\langle\boldsymbol{\beta}+\boldsymbol{B} \boldsymbol{x}, \boldsymbol{f}^{\prime}(\boldsymbol{x})\right\rangle+\int_{U_{d}}(f(\boldsymbol{x}+\boldsymbol{z})-f(\boldsymbol{x})) \nu(\mathrm{d} \boldsymbol{z}) \\
& +\sum_{i=1}^{d} x_{i} \int_{U_{d}}\left(f(\boldsymbol{x}+\boldsymbol{z})-f(\boldsymbol{x})-f_{i}^{\prime}(\boldsymbol{x})\left(1 \wedge z_{i}\right)\right) \mu_{i}(\mathrm{~d} \boldsymbol{z})
\end{aligned}
$$

for $f \in C_{\mathrm{c}}^{2}\left(\mathbb{R}_{+}^{d}, \mathbb{R}\right)$ and $\boldsymbol{x} \in \mathbb{R}_{+}^{d}$, where $f_{i}^{\prime}$ and $f_{i, i}^{\prime \prime}, \quad i \in\{1, \ldots, d\}$, denote the first and second order partial derivatives of $f$ with respect to its $i$-th variable, respectively, and $\boldsymbol{f}^{\prime}(\boldsymbol{x}):=\left(f_{1}^{\prime}(\boldsymbol{x}), \ldots, f_{d}^{\prime}(\boldsymbol{x})\right)^{\top}$. 
2.5 Remark. This theorem is a special case of Theorem 2.7 of Duffie et al. 9] with $m=d$, $n=0$ and zero killing rate.

2.6 Definition. A conservative Markov process with state space $\mathbb{R}_{+}^{d}$ and with transition semigroup $\left(P_{t}\right)_{t \in \mathbb{R}_{+}}$given in Theorem 2.4 is called a multi-type CBI process with parameters $(d, \boldsymbol{c}, \boldsymbol{\beta}, \boldsymbol{B}, \nu, \boldsymbol{\mu})$.

Let $\left(\boldsymbol{X}_{t}\right)_{t \in \mathbb{R}_{+}}$be a multi-type CBI process with parameters $(d, \boldsymbol{c}, \boldsymbol{\beta}, \boldsymbol{B}, \nu, \boldsymbol{\mu})$ such that $\mathbb{E}\left(\left\|\boldsymbol{X}_{0}\right\|\right)<\infty$ and the moment condition

$$
\int_{U_{d}}\|\boldsymbol{z}\| \mathbb{1}_{\{\|\boldsymbol{z}\| \geqslant 1\}} \nu(\mathrm{d} \boldsymbol{z})<\infty
$$

holds. Then, by Lemma 3.4 in Barczy et al. [4],

$$
\mathbb{E}\left(\boldsymbol{X}_{t}\right)=\mathrm{e}^{t \widetilde{\boldsymbol{B}}} \mathbb{E}\left(\boldsymbol{X}_{0}\right)+\left(\int_{0}^{t} \mathrm{e}^{u \widetilde{\boldsymbol{B}}} \mathrm{d} u\right) \widetilde{\boldsymbol{\beta}}, \quad t \in \mathbb{R}_{+},
$$

where

$$
\begin{gathered}
\widetilde{\boldsymbol{B}}:=\left(\widetilde{b}_{i, j}\right)_{i, j \in\{1, \ldots, d\}}, \quad \widetilde{b}_{i, j}:=b_{i, j}+\int_{U_{d}}\left(z_{i}-\delta_{i, j}\right)^{+} \mu_{j}(\mathrm{~d} \boldsymbol{z}), \\
\widetilde{\boldsymbol{\beta}}:=\boldsymbol{\beta}+\int_{U_{d}} \boldsymbol{z} \nu(\mathrm{d} \boldsymbol{z}),
\end{gathered}
$$

with $\delta_{i, j}:=1$ if $i=j$, and $\delta_{i, j}:=0$ if $i \neq j$. Note that $\widetilde{\boldsymbol{B}} \in \mathbb{R}_{(+)}^{d \times d}$ and $\widetilde{\boldsymbol{\beta}} \in \mathbb{R}_{+}^{d}$, since

$$
\int_{U_{d}}\|\boldsymbol{z}\| \nu(\mathrm{d} \boldsymbol{z})<\infty, \quad \int_{U_{d}}\left(z_{i}-\delta_{i, j}\right)^{+} \mu_{j}(\mathrm{~d} \boldsymbol{z})<\infty, \quad i, j \in\{1, \ldots, d\},
$$

see Barczy et al. [4, Section 2].

\section{Classification of multi-type CBI processes and mo- ment estimations}

For a matrix $\boldsymbol{A} \in \mathbb{R}^{d \times d}, \sigma(\boldsymbol{A})$ will denote the spectrum of $\boldsymbol{A}$, i.e., the set of the eigenvalues of $\boldsymbol{A}$. Then $r(\boldsymbol{A}):=\max _{\lambda \in \sigma(\boldsymbol{A})}|\lambda|$ is the spectral radius of $\boldsymbol{A}$. Moreover, we will use the notation

$$
s(\boldsymbol{A}):=\max _{\lambda \in \sigma(\boldsymbol{A})} \operatorname{Re}(\lambda) .
$$

By the spectral mapping theorem (see, e.g., Dunford and Schwartz [10, Theorem VII.3.11]), $\sigma\left(\mathrm{e}^{t \boldsymbol{A}}\right)=\mathrm{e}^{t \sigma(\boldsymbol{A})}$ for all $t \in \mathbb{R}_{+}$. Consequently,

$$
r\left(\mathrm{e}^{t \boldsymbol{A}}\right)=\max _{\lambda \in \sigma(\boldsymbol{A})}\left|\mathrm{e}^{t \lambda}\right|=\max _{\lambda \in \sigma(\boldsymbol{A})} \mathrm{e}^{t \operatorname{Re}(\lambda)}=\mathrm{e}^{s(\boldsymbol{A}) t}, \quad t \in \mathbb{R}_{+}
$$


and hence $s(\boldsymbol{A})=\log r\left(\mathrm{e}^{\boldsymbol{A}}\right)$. A matrix $\boldsymbol{A} \in \mathbb{R}^{d \times d}$ is called reducible if there exist a permutation matrix $\boldsymbol{P} \in \mathbb{R}^{d \times d}$ and an integer $r$ with $1 \leqslant r \leqslant d-1$ such that

$$
\boldsymbol{P}^{\top} \boldsymbol{A P}=\left[\begin{array}{cc}
\boldsymbol{A}_{1} & \boldsymbol{A}_{2} \\
\mathbf{0} & \boldsymbol{A}_{3}
\end{array}\right],
$$

where $\boldsymbol{A}_{1} \in \mathbb{R}^{r \times r}, \quad \boldsymbol{A}_{3} \in \mathbb{R}^{(d-r) \times(d-r)}, \quad \boldsymbol{A}_{2} \in \mathbb{R}^{r \times(d-r)}$, and $\mathbf{0} \in \mathbb{R}^{(d-r) \times r}$ is a null matrix. A matrix $\boldsymbol{A} \in \mathbb{R}^{d \times d}$ is called irreducible if it is not reducible, see, e.g., Horn and Johnson [12, Definitions 6.2.21 and 6.2.22]. We do emphasize that no 1-by-1 matrix is reducible.

If $\left(\boldsymbol{X}_{t}\right)_{t \in \mathbb{R}_{+}}$is a CBI process with parameters $(d, \boldsymbol{c}, \boldsymbol{\beta}, \boldsymbol{B}, \nu, \boldsymbol{\mu})$ such that the moment condition (2.3) holds, then $\mathrm{e}^{t \widetilde{\boldsymbol{B}}} \in \mathbb{R}_{+}^{d \times d}$ for all $t \in \mathbb{R}_{+}$, since $\widetilde{\boldsymbol{B}} \in \mathbb{R}_{(+)}^{d \times d}$, see the explanation before Lemma A.1. Moreover, by Lemma A.1 and Remark A.2, $\mathrm{e}^{t_{0} \widetilde{\boldsymbol{B}}} \in \mathbb{R}_{++}^{d \times d}$ for some (and hence for all) $t_{0} \in \mathbb{R}_{++}$if and only if $\widetilde{\boldsymbol{B}}$ is irreducible or, if and only if $\mathrm{e}^{\widetilde{\boldsymbol{B}}}$ is irreducible.

3.1 Definition. Let $\left(\boldsymbol{X}_{t}\right)_{t \in \mathbb{R}_{+}}$be a multi-type CBI process with parameters $(d, \boldsymbol{c}, \boldsymbol{\beta}, \boldsymbol{B}, \nu, \boldsymbol{\mu})$ such that the moment condition (2.3) holds. Then $\left(\boldsymbol{X}_{t}\right)_{t \in \mathbb{R}_{+}}$is called irreducible if $\widetilde{\boldsymbol{B}}$ is irreducible.

Next we introduce a classification of irreducible multi-type CBI processes. Formula (2.4) shows that the semigroup $\left(\mathrm{e}^{t \widetilde{\boldsymbol{B}}}\right)_{t \in \mathbb{R}_{+}}$of matrices plays a crucial role in the asymptotic behavior of the expectations $\mathbb{E}\left(\boldsymbol{X}_{t}\right)$ as $t \rightarrow \infty$ described in Proposition B.1. This gives a motivation for a classification of irreducible multi-type CBI processes.

3.2 Definition. Let $\left(\boldsymbol{X}_{t}\right)_{t \in \mathbb{R}_{+}}$be a multi-type $C B I$ process with parameters $(d, \boldsymbol{c}, \boldsymbol{\beta}, \boldsymbol{B}, \nu, \boldsymbol{\mu})$ such that $\mathbb{E}\left(\left\|\boldsymbol{X}_{0}\right\|\right)<\infty$ and the moment condition (2.3) holds. Suppose that $\left(\boldsymbol{X}_{t}\right)_{t \in \mathbb{R}_{+}}$is irreducible. Then $\left(\boldsymbol{X}_{t}\right)_{t \in \mathbb{R}_{+}}$is called

$$
\begin{cases}\text { subcritical } & \text { if } s(\widetilde{\boldsymbol{B}})<0, \\ \text { critical } & \text { if } s(\widetilde{\boldsymbol{B}})=0, \\ \text { supercritical } & \text { if } s(\widetilde{\boldsymbol{B}})>0 .\end{cases}
$$

The classification for subcritical, critical and supercritical cases in Definition 3.2 is in accordance with the corresponding classification for single-type continuous state and continuous time branching processes, see, e.g., Li [23, page 58].

\section{Convergence result}

A function $f: \mathbb{R}_{+} \rightarrow \mathbb{R}^{d}$ is called càdlàg if it is right continuous with left limits. Let $\mathbb{D}\left(\mathbb{R}_{+}, \mathbb{R}^{d}\right)$ and $\mathbb{C}\left(\mathbb{R}_{+}, \mathbb{R}^{d}\right)$ denote the space of all $\mathbb{R}^{d}$-valued càdlàg and continuous functions on $\mathbb{R}_{+}$, respectively. Let $\mathcal{D}_{\infty}\left(\mathbb{R}_{+}, \mathbb{R}^{d}\right)$ denote the Borel $\sigma$-field in $\mathbb{D}\left(\mathbb{R}_{+}, \mathbb{R}^{d}\right)$ for the metric characterized by Jacod and Shiryaev [19, VI.1.15] (with this metric, $\mathbb{D}\left(\mathbb{R}_{+}, \mathbb{R}^{d}\right.$ ) is a complete 
and separable metric space). For $\mathbb{R}^{d}$-valued stochastic processes $\left(\mathcal{Y}_{t}\right)_{t \in \mathbb{R}_{+}}$and $\left(\mathcal{Y}_{t}^{n}\right)_{t \in \mathbb{R}_{+}}$, $n \in \mathbb{N}$, with càdlàg paths we write $\mathcal{Y}^{n} \stackrel{\mathcal{D}}{\longrightarrow} \mathcal{Y}$ as $n \rightarrow \infty$ if the distribution of $\mathcal{Y}^{n}$ on the space $\left(\mathbb{D}\left(\mathbb{R}_{+}, \mathbb{R}^{d}\right), \mathcal{D}_{\infty}\left(\mathbb{R}_{+}, \mathbb{R}^{d}\right)\right)$ converges weakly to the distribution of $\mathcal{Y}$ on the space $\left(\mathbb{D}\left(\mathbb{R}_{+}, \mathbb{R}^{d}\right), \mathcal{D}_{\infty}\left(\mathbb{R}_{+}, \mathbb{R}^{d}\right)\right)$ as $n \rightarrow \infty$.

4.1 Theorem. Let $\left(\boldsymbol{X}_{t}\right)_{t \in \mathbb{R}_{+}}$be a multi-type CBI process with parameters $(d, \boldsymbol{c}, \boldsymbol{\beta}, \boldsymbol{B}, \nu, \boldsymbol{\mu})$ such that $\mathbb{E}\left(\left\|\boldsymbol{X}_{0}\right\|^{4}\right)<\infty$ and

$$
\int_{U_{d}}\|\boldsymbol{z}\|^{4} \mathbb{1}_{\{\|\boldsymbol{z}\| \geqslant 1\}} \nu(\mathrm{d} \boldsymbol{z})<\infty, \quad \int_{U_{d}}\|\boldsymbol{z}\|^{4} \mathbb{1}_{\{\|\boldsymbol{z}\| \geqslant 1\}} \mu_{i}(\mathrm{~d} \boldsymbol{z})<\infty, \quad i \in\{1, \ldots, d\} .
$$

Suppose that $\left(\boldsymbol{X}_{t}\right)_{t \in \mathbb{R}_{+}}$is irreducible and critical. Then

$$
\left(\mathcal{X}_{t}^{(n)}\right)_{t \in \mathbb{R}_{+}}:=\left(n^{-1} \boldsymbol{X}_{\lfloor n t\rfloor}\right)_{t \in \mathbb{R}_{+}} \stackrel{\mathcal{D}}{\longrightarrow}\left(\mathcal{X}_{t}\right)_{t \in \mathbb{R}_{+}}:=\left(\mathcal{X}_{t} \boldsymbol{u}\right)_{t \in \mathbb{R}_{+}} \quad \text { as } n \rightarrow \infty
$$

in $\mathbb{D}\left(\mathbb{R}_{+}, \mathbb{R}^{d}\right)$, where $\boldsymbol{u}:=\boldsymbol{u}_{\widetilde{\boldsymbol{B}}} \in \mathbb{R}_{++}^{d}$ is the right Perron vector of $\mathrm{e}^{\widetilde{\boldsymbol{B}}}$ corresponding to the eigenvalue 1 with $\sum_{i=1}^{d} \boldsymbol{e}_{i}^{\top} \boldsymbol{u}=1$ (see (ii) of Lemma A.3), ( $\left.\mathcal{X}_{t}\right)_{t \in \mathbb{R}_{+}}$is the unique strong solution of the SDE

$$
\mathrm{d} \mathcal{X}_{t}=\langle\boldsymbol{v}, \widetilde{\boldsymbol{\beta}}\rangle \mathrm{d} t+\sqrt{\langle\overline{\boldsymbol{C}} \boldsymbol{v}, \boldsymbol{v}\rangle \mathcal{X}_{t}^{+}} \mathrm{d} \mathcal{W}_{t}, \quad t \in \mathbb{R}_{+}, \quad \mathcal{X}_{0}=0,
$$

where $\boldsymbol{v}:=\boldsymbol{v}_{\widetilde{\boldsymbol{B}}} \in \mathbb{R}_{++}^{d}$ is the left Perron vector of $\mathrm{e}^{\widetilde{\boldsymbol{B}}}$ corresponding to the eigenvalue 1 with $\boldsymbol{v}^{\top} \boldsymbol{u}=1$ (see (iii) of Lemma A.3), ( $\left.\mathcal{W}_{t}\right)_{t \in \mathbb{R}_{+}}$is a standard Brownian motion, $\widetilde{\boldsymbol{\beta}}$ is given in (2.6), and

$$
\overline{\boldsymbol{C}}:=\sum_{k=1}^{d}\left\langle\boldsymbol{e}_{k}, \boldsymbol{u}\right\rangle \boldsymbol{C}_{k} \in \mathbb{R}_{+}^{d \times d}
$$

with

$$
\boldsymbol{C}_{k}:=2 c_{k} \boldsymbol{e}_{k} \boldsymbol{e}_{k}^{\top}+\int_{U_{d}} \boldsymbol{z} \boldsymbol{z}^{\top} \mu_{k}(\mathrm{~d} \boldsymbol{z}) \in \mathbb{R}_{+}^{d \times d}, \quad k \in\{1, \ldots, d\}
$$

4.2 Remark. We suspect that the moment conditions might be relaxed to $\mathbb{E}\left(\left\|\boldsymbol{X}_{0}\right\|^{2}\right)<\infty$ and

$$
\int_{U_{d}}\|\boldsymbol{z}\|^{2} \mathbb{1}_{\{\|\boldsymbol{z}\| \geqslant 1\}} \nu(\mathrm{d} \boldsymbol{z})<\infty, \quad \int_{U_{d}}\|\boldsymbol{z}\|^{2} \mathbb{1}_{\{\|\boldsymbol{z}\| \geqslant 1\}} \mu_{i}(\mathrm{~d} \boldsymbol{z})<\infty, \quad i \in\{1, \ldots, d\} .
$$

In fact, the higher order moment assumptions are used only for checking the conditional Lindeberg condition, namely, condition (ii) of Theorem C.1, in order to prove convergence (6.3) of an appropriately defined sequence of martingale differences (6.2). One might check the conditional Lindeberg condition under $\mathbb{E}\left(\left\|\boldsymbol{X}_{0}\right\|^{2}\right)<\infty$ and the above weaker moment assumptions (4.6) by the method of Ispány and Pap [15], see also this method in Barczy et al. [3, proof of convergence (5.2)]. One might generalize Theorem 4.1 to an appropriate sequence of initial distributions instead of a fixed one, see, e.g., the method of Ispány and Pap [17]. 
4.3 Remark. Among the moment conditions we have the relationships (4.1) $\Rightarrow(4.6) \Rightarrow(2.3)$. The moment conditions (4.6) together with the fact that $\nu$ and $\boldsymbol{\mu}$ satisfy Definition 2.2 imply

$$
\int_{U_{d}}\|\boldsymbol{z}\|^{2} \nu(\mathrm{d} \boldsymbol{z})<\infty, \quad \int_{U_{d}}\|\boldsymbol{z}\|^{2} \mu_{k}(\mathrm{~d} \boldsymbol{z})<\infty, \quad k \in\{1, \ldots, d\} .
$$

Indeed,

$$
\begin{aligned}
\int_{U_{d}}\|\boldsymbol{z}\|^{2} \nu(\mathrm{d} \boldsymbol{z}) & =\int_{U_{d}}\|\boldsymbol{z}\|^{2} \mathbb{1}_{\{\|\boldsymbol{z}\|<1\}} \nu(\mathrm{d} \boldsymbol{z})+\int_{U_{d}}\|\boldsymbol{z}\|^{2} \mathbb{1}_{\{\|\boldsymbol{z}\| \geqslant 1\}} \nu(\mathrm{d} \boldsymbol{z}) \\
& \leqslant \int_{U_{d}}(1 \wedge\|\boldsymbol{z}\|) \nu(\mathrm{d} \boldsymbol{z})+\int_{U_{d}}\|\boldsymbol{z}\|^{2} \mathbb{1}_{\{\|\boldsymbol{z}\| \geqslant 1\}} \nu(\mathrm{d} \boldsymbol{z})<\infty
\end{aligned}
$$

and for all $k \in\{1, \ldots, d\}$,

$$
\begin{aligned}
\int_{U_{d}}\|\boldsymbol{z}\|^{2} \mu_{k}(\mathrm{~d} \boldsymbol{z}) & =\int_{U_{d}}\|\boldsymbol{z}\|^{2} \mathbb{1}_{\{\|\boldsymbol{z}\|<1\}} \mu_{k}(\mathrm{~d} \boldsymbol{z})+\int_{U_{d}}\|\boldsymbol{z}\|^{2} \mathbb{1}_{\{\|\boldsymbol{z}\| \geqslant 1\}} \mu_{k}(\mathrm{~d} \boldsymbol{z}) \\
& \leqslant \int_{U_{d}}\|\boldsymbol{z}\| \wedge\|\boldsymbol{z}\|^{2} \mu_{k}(\mathrm{~d} \boldsymbol{z})+\int_{U_{d}}\|\boldsymbol{z}\|^{2} \mathbb{1}_{\{\|\boldsymbol{z}\| \geqslant 1\}} \mu_{k}(\mathrm{~d} \boldsymbol{z})<\infty .
\end{aligned}
$$

Clearly, (4.7) implies also $\boldsymbol{C}_{k} \in \mathbb{R}_{+}^{d \times d}, k \in\{1, \ldots, d\}$, since

$$
\int_{U_{d}}\left\|\boldsymbol{z} \boldsymbol{z}^{\top}\right\| \mu_{k}(\mathrm{~d} \boldsymbol{z}) \leqslant \int_{U_{d}}\|\boldsymbol{z}\|^{2} \mu_{k}(\mathrm{~d} \boldsymbol{z})<\infty .
$$

Obviously, $\boldsymbol{C}_{k}, \quad k \in\{1, \ldots, d\}$, and $\overline{\boldsymbol{C}}$ are symmetric positive semidefinite matrices, and $\overline{\boldsymbol{C}}=\mathbf{0}$ if and only if $\boldsymbol{C}_{k}=\mathbf{0}$ for all $k \in\{1, \ldots, d\}$. Indeed, $\overline{\boldsymbol{C}}=\mathbf{0}$ implies

$$
0=\left\langle\overline{\boldsymbol{C}} \boldsymbol{e}_{i}, \boldsymbol{e}_{i}\right\rangle=\sum_{k=1}^{d} \boldsymbol{e}_{k}^{\top} \boldsymbol{u}\left\langle\boldsymbol{C}_{k} \boldsymbol{e}_{i}, \boldsymbol{e}_{i}\right\rangle, \quad i \in\{1, \ldots, d\},
$$

and, since $\boldsymbol{e}_{k}^{\top} \boldsymbol{u} \in \mathbb{R}_{++}$and $\left\langle\boldsymbol{C}_{k} \boldsymbol{e}_{i}, \boldsymbol{e}_{i}\right\rangle \in \mathbb{R}_{+}$(due to positive semidefiniteness of $\boldsymbol{C}_{k}$ ), we get $\left\langle\boldsymbol{C}_{k} \boldsymbol{e}_{i}, \boldsymbol{e}_{i}\right\rangle=0, k, i \in\{1, \ldots, d\}$. For each $k, i \in\{1, \ldots, d\}$, we have $0=\left\langle\boldsymbol{C}_{k} \boldsymbol{e}_{i}, \boldsymbol{e}_{i}\right\rangle=$ $\left\langle\sqrt{\boldsymbol{C}_{k}} \boldsymbol{e}_{i}, \sqrt{\boldsymbol{C}_{k}} \boldsymbol{e}_{i}\right\rangle=\left\|\sqrt{\boldsymbol{C}_{k}} \boldsymbol{e}_{i}\right\|^{2}$, where $\sqrt{\boldsymbol{C}_{k}}$ denotes the unique symmetric and positive semidefinite square root of $\boldsymbol{C}_{k}$. Consequently, $\sqrt{\boldsymbol{C}_{k}} \boldsymbol{e}_{i}=\mathbf{0}$, thus $\boldsymbol{C}_{k} \boldsymbol{e}_{i}=\sqrt{\boldsymbol{C}_{k}} \sqrt{\boldsymbol{C}_{k}} \boldsymbol{e}_{i}=\mathbf{0}$, implying $\left\langle\boldsymbol{C}_{k} \boldsymbol{e}_{i}, \boldsymbol{e}_{j}\right\rangle=0$ for each $k, i, j \in\{1, \ldots, d\}$. Hence $\overline{\boldsymbol{C}}=\mathbf{0}$ if and only if $c_{k}=0$ and $\mu_{k}=0$ for all $k \in\{1, \ldots, d\}$.

4.4 Remark. The SDE (4.3) has a pathwise unique strong solution $\left(\mathcal{X}_{t}^{(x)}\right)_{t \in \mathbb{R}_{+}}$for all initial values $\mathcal{X}_{0}^{(x)}=x \in \mathbb{R}$, and if the initial value $x$ is nonnegative, then $\mathcal{X}_{t}^{(x)}$ is nonnegative for all $t \in \mathbb{R}_{+}$with probability one, since $\langle\boldsymbol{v}, \widetilde{\boldsymbol{\beta}}\rangle \in \mathbb{R}_{+}$. In fact, $\left(4\langle\overline{\boldsymbol{C}} \boldsymbol{v}, \boldsymbol{v}\rangle^{-1} \mathcal{X}_{t}^{(x)}\right)_{t \in \mathbb{R}_{+}}$is a square of a $4\langle\overline{\boldsymbol{C}} \boldsymbol{v}, \boldsymbol{v}\rangle^{-1}\langle\boldsymbol{v}, \widetilde{\boldsymbol{\beta}}\rangle$-dimensional Bessel process started at $x$ (see, e.g., Revuz and Yor [27, Definitions XI.1.1]). Moreover, if $\langle\boldsymbol{v}, \widetilde{\boldsymbol{\beta}}\rangle>0$ then $\mathbb{P}\left(\mathcal{X}_{t}>0\right)=1$ for all $t>0$, where $\mathcal{X}_{t}=\mathcal{X}_{t}^{(0)}, t \in \mathbb{R}_{+}$. Indeed, we have $\mathcal{X}_{t}=\langle\boldsymbol{v}, \widetilde{\boldsymbol{\beta}}\rangle t, t \in \mathbb{R}_{+}$, if $\langle\overline{\boldsymbol{C}} \boldsymbol{v}, \boldsymbol{v}\rangle=0$, and $\mathcal{X}_{t}$ has a gamma distribution with parameters $2\langle\boldsymbol{v}, \widetilde{\boldsymbol{\beta}}\rangle /\langle\overline{\boldsymbol{C}} \boldsymbol{v}, \boldsymbol{v}\rangle$ and $2 /(\langle\overline{\boldsymbol{C}} \boldsymbol{v}, \boldsymbol{v}\rangle t)$ if $\langle\overline{\boldsymbol{C}} \boldsymbol{v}, \boldsymbol{v}\rangle \neq 0$ and $t>\underset{\widetilde{\beta}}{0}$. For the proofs, see, e.g., Ikeda and Watanabe [14, Chapter IV, Example 8.2]. Note that $\langle\boldsymbol{v}, \widetilde{\boldsymbol{\beta}}\rangle>0$ if and only if $\widetilde{\boldsymbol{\beta}} \neq \mathbf{0}$, which is equivalent to $\boldsymbol{\beta} \neq \mathbf{0}$ or $\nu \neq 0$. 
In the next remark we specialize Theorem 4.1 for dimension 1.

4.5 Remark. If $\left(X_{t}\right)_{t \in \mathbb{R}_{+}}$is a single-type (1-dimensional and hence irreducible) critical CBI process with parameters $(1, c, \beta, b, \nu, \mu)$ satisfying assumptions of Theorem 4.1, then $\tilde{b}:=$ $b+\int_{1}^{\infty}(z-1) \mu(\mathrm{d} z)=0$ (due to criticality and using that $s(\widetilde{b})=\widetilde{b}$ in dimension 1 ), and $\left(n^{-1} X_{\lfloor n t\rfloor}\right)_{t \in \mathbb{R}_{+}} \stackrel{\mathcal{D}}{\longrightarrow}\left(\mathcal{X}_{t}\right)_{t \in \mathbb{R}_{+}}$as $n \rightarrow \infty$, where $\left(\mathcal{X}_{t}\right)_{t \in \mathbb{R}_{+}}$is a pathwise unique strong solution of the $\mathrm{SDE} \mathrm{d} \mathcal{X}_{t}=\widetilde{\beta} \mathrm{d} t+\sqrt{\bar{C} \mathcal{X}_{t}^{+}} \mathrm{d} \mathcal{W}_{t}, \quad t \in \mathbb{R}_{+}$, with initial value $\mathcal{X}_{0}=0$ and with $\widetilde{\beta}:=\beta+\int_{U_{1}} z \nu(\mathrm{d} z), \quad \bar{C}:=2 c+\int_{U_{1}} z^{2} \mu(\mathrm{d} z)$. Here, by (2.2), the infinitesimal generator of $\left(X_{t}\right)_{t \in \mathbb{R}_{+}}$is

$$
\begin{aligned}
\left(\mathcal{A}_{X} f\right)(x)= & c x f^{\prime \prime}(x)+(\beta+b x) f^{\prime}(x)+\int_{U_{1}}(f(x+z)-f(x)) \nu(\mathrm{d} z) \\
& +x \int_{U_{1}}\left(f(x+z)-f(x)-f^{\prime}(x)(1 \wedge z)\right) \mu(\mathrm{d} z) \\
= & \frac{1}{2} \bar{C} x f^{\prime \prime}(x)+(\widetilde{\beta}+b x) f^{\prime}(x)+\int_{U_{1}}\left(f(x+z)-f(x)-f^{\prime}(x) z\right) \nu(\mathrm{d} z) \\
& +x \int_{U_{1}}\left(f(x+z)-f(x)-f^{\prime}(x)(1 \wedge z)-\frac{1}{2} f^{\prime \prime}(x) z^{2}\right) \mu(\mathrm{d} z) \\
= & \frac{1}{2} \bar{C} x f^{\prime \prime}(x)+\widetilde{\beta} f^{\prime}(x)+\int_{U_{1}}\left(f(x+z)-f(x)-f^{\prime}(x) z\right) \nu(\mathrm{d} z) \\
& +x \int_{U_{1}}\left(f(x+z)-f(x)-f^{\prime}(x) z-\frac{1}{2} f^{\prime \prime}(x) z^{2}\right) \mu(\mathrm{d} z)
\end{aligned}
$$

for $f \in \mathbb{C}_{\mathrm{c}}^{2}\left(\mathbb{R}_{+}, \mathbb{R}\right)$ and $x \in \mathbb{R}_{+}$, where the last equality follows by

$$
b+\int_{U_{1}}(z-(1 \wedge z)) \mu(\mathrm{d} z)=b+\int_{1}^{\infty}(z-1) \mu(\mathrm{d} z)=\widetilde{b}=0 .
$$

Further, the limit process $\left(\mathcal{X}_{t}\right)_{t \in \mathbb{R}_{+}}$is a single-type (1-dimensional) CBI diffusion process with parameters $\left(1, \frac{1}{2} \bar{C}, \widetilde{\beta}, 0,0,0\right)$, and its infinitesimal generator takes the form

$$
\left(\mathcal{A}_{\mathcal{X}} f\right)(x)=\frac{1}{2} \bar{C} x f^{\prime \prime}(x)+\widetilde{\beta} f^{\prime}(x)
$$

for $f \in \mathbb{C}_{\mathrm{c}}^{2}\left(\mathbb{R}_{+}, \mathbb{R}\right)$ and $x \in \mathbb{R}_{+}$, see, e.g., Karatzas and Shreve [22, Section 5.1]. Note that under the conditions

$$
\int_{U_{1}} z \nu(\mathrm{d} z)<\infty, \quad \int_{U_{1}} z^{2} \mu(\mathrm{d} z)<\infty,
$$

a stronger statement, namely, a scaling limit theorem $\left(n^{-1} X_{n t}\right)_{t \in \mathbb{R}_{+}} \stackrel{\mathcal{D}}{\longrightarrow}\left(\mathcal{X}_{t}\right)_{t \in \mathbb{R}_{+}}$is also valid, see Barczy et al. [2, Corollary 2.1] (under the additional stronger assumption $\int_{U_{1}} z^{2} \nu(\mathrm{d} z)<\infty$, Huang et al. [13, Theorem 2.3] proved this result with another method).

In the next remark we point out that in case of $d \geqslant 2$, the limit process $\left(\boldsymbol{\mathcal { X }}_{t}\right)_{t \in \mathbb{R}_{+}}$in (4.2) is not a $d$-type ( $d$-dimensional) CBI process. 
4.6 Remark. If $d \geqslant 2$ and $\left(\boldsymbol{X}_{t}\right)_{t \in \mathbb{R}_{+}}$is an irreducible and critical $d$-type CBI process with parameters $(d, \boldsymbol{c}, \boldsymbol{\beta}, \boldsymbol{B}, \nu, \boldsymbol{\mu})$ satisfying assumptions of Theorem 4.1, then the limit process $\left(\mathcal{X}_{t}\right)_{t \in \mathbb{R}_{+}}$is not a $d$-type CBI process, i.e., in case $d \geqslant 2$, we have a different limit behaviour compared to dimension 1 , see Remark 4.5. Indeed, $\left(\boldsymbol{\mathcal { X }}_{t}\right)_{t \in \mathbb{R}_{+}}$is a time homogeneous Markov process with state space $\mathbb{R}_{+} \boldsymbol{u}$ and with infinitesimal generator

$$
\left(\mathcal{A}_{\mathcal{X}} f\right)(\boldsymbol{x})=\frac{1}{2} \boldsymbol{v}^{\top} \overline{\boldsymbol{C}} \boldsymbol{v} x \widetilde{f}^{\prime \prime}(x)+\boldsymbol{v}^{\top} \widetilde{\boldsymbol{\beta}} \widetilde{f}^{\prime}(x), \quad \boldsymbol{x}=x \boldsymbol{u}, \quad x \in \mathbb{R}_{+}
$$

for $f: \mathbb{R}_{+} \boldsymbol{u} \rightarrow \mathbb{R}, \quad f(x \boldsymbol{u})=\tilde{f}(x)$ with $\tilde{f} \in \mathbb{C}_{\mathrm{c}}^{2}\left(\mathbb{R}_{+}, \mathbb{R}\right)$, since

$$
\begin{aligned}
\left(\mathcal{A}_{\mathcal{X}} f\right)(\boldsymbol{x}) & =\lim _{h \downarrow 0} h^{-1}\left[\mathbb{E}\left(f\left(\mathcal{X}_{h}\right) \mid \mathcal{X}_{0}=\boldsymbol{x}\right)-f(\boldsymbol{x})\right]=\lim _{h \downarrow 0} h^{-1}\left[\mathbb{E}\left(f\left(\mathcal{X}_{h} \boldsymbol{u}\right) \mid \mathcal{X}_{0} \boldsymbol{u}=x \boldsymbol{u}\right)-f(x \boldsymbol{u})\right] \\
& =\lim _{h \downarrow 0} h^{-1}\left[\mathbb{E}\left(\tilde{f}\left(\mathcal{X}_{h}\right) \mid \mathcal{X}_{0}=x\right)-\widetilde{f}(x)\right]=\left(\mathcal{A}_{\mathcal{X}} \widetilde{f}\right)(x)
\end{aligned}
$$

has the above form by (4.3), see, e.g., Karatzas and Shreve [22, Section 5.1]. Clearly, the infinitesimal generator $\mathcal{A}_{\mathcal{X}}$ is not of the form (2.2), since it is not defined for all $f \in \mathbb{C}_{\mathrm{c}}^{2}\left(\mathbb{R}_{+}^{d}, \mathbb{R}\right)$ due to $d \geqslant 2$. Note that the process $\left(\mathcal{X}_{t}\right)_{t \in \mathbb{R}_{+}}$is a single-type (one-dimensional) CBI process with parameters $\left(1, \frac{1}{2} \boldsymbol{v}^{\top} \overline{\boldsymbol{C}} \boldsymbol{v}, \boldsymbol{v}^{\top} \widetilde{\boldsymbol{\beta}}, 0,0,0\right)$.

Next we formulate a corollary of Theorem 4.1 deriving a limit distribution for the relative frequencies of distinct types of individuals.

4.7 Corollary. Assume that the conditions of Theorem 4.1 holds. If, in addition, $\boldsymbol{\beta} \neq \mathbf{0}$ or $\nu \neq 0$, then for each $t>0$ and $i, j \in\{1, \ldots, d\}$,

$$
\frac{\boldsymbol{e}_{i}^{\top} \boldsymbol{X}_{\lfloor n t\rfloor}}{\boldsymbol{e}_{j}^{\top} \boldsymbol{X}_{\lfloor n t\rfloor}} \stackrel{\mathbb{P}}{\longrightarrow} \frac{\boldsymbol{e}_{i}^{\top} \boldsymbol{u}}{\boldsymbol{e}_{j}^{\top} \boldsymbol{u}} \quad \text { and } \quad \frac{\boldsymbol{e}_{i}^{\top} \boldsymbol{X}_{\lfloor n t\rfloor}}{\sum_{k=1}^{d} \boldsymbol{e}_{k}^{\top} \boldsymbol{X}_{\lfloor n t\rfloor}} \stackrel{\mathbb{P}}{\longrightarrow} \boldsymbol{e}_{i}^{\top} \boldsymbol{u} \quad \text { as } n \rightarrow \infty .
$$

Proof. Theorem 4.1 implies $\left(\boldsymbol{e}_{i}^{\top} \boldsymbol{X}_{\lfloor n t\rfloor}, \boldsymbol{e}_{j}^{\top} \boldsymbol{X}_{\lfloor n t\rfloor}\right) \stackrel{\mathcal{D}}{\longrightarrow}\left(\boldsymbol{e}_{i}^{\top} \boldsymbol{u} \mathcal{X}_{t}, \boldsymbol{e}_{j}^{\top} \boldsymbol{u} \mathcal{X}_{t}\right)$ as $n \rightarrow \infty$ for each $t \in \mathbb{R}_{+}$. The function $g: \mathbb{R}^{2} \rightarrow \mathbb{R}$, defined by

$$
g(x, y):= \begin{cases}\frac{x}{y}, & \text { if } x \in \mathbb{R} \text { and } y \neq 0 \\ 0, & \text { if } x \in \mathbb{R} \text { and } y=0\end{cases}
$$

is continuous on the set $\mathbb{R} \times(\mathbb{R} \backslash\{0\})$, and the distribution of $\left(\boldsymbol{e}_{i}^{\top} \boldsymbol{u} \mathcal{X}_{t}, \boldsymbol{e}_{j}^{\top} \boldsymbol{u} \mathcal{X}_{t}\right)$ is concentrated on this set, since, by Remark 4.4. $\mathbb{P}\left(\mathcal{X}_{t}>0\right)=1$, and $\boldsymbol{e}_{j}^{\top} \boldsymbol{u}>0$. Hence the continuous mapping theorem yields that

$$
g\left(\boldsymbol{e}_{i}^{\top} \boldsymbol{X}_{\lfloor n t\rfloor}, \boldsymbol{e}_{j}^{\top} \boldsymbol{X}_{\lfloor n t\rfloor}\right) \stackrel{\mathcal{D}}{\longrightarrow} g\left(\boldsymbol{e}_{i}^{\top} \boldsymbol{u} \mathcal{X}_{t}, \boldsymbol{e}_{j}^{\top} \boldsymbol{u} \mathcal{X}_{t}\right)=\frac{\boldsymbol{e}_{i}^{\top} \boldsymbol{u}}{\boldsymbol{e}_{j}^{\top} \boldsymbol{u}} \quad \text { as } n \rightarrow \infty,
$$

thus we obtain the first convergence. Moreover,

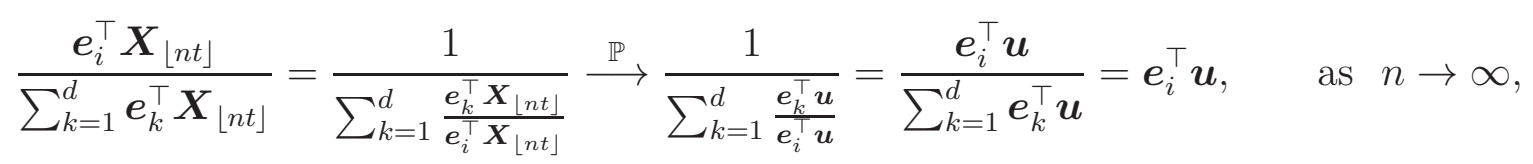

hence we obtain the second convergence. 


\section{$5 \quad$ Examples}

In this section we give some examples for multi-type CBI processes with parameters $(d, \boldsymbol{c}, \boldsymbol{\beta}, \boldsymbol{B}, \nu, \boldsymbol{\mu})$ for which we give the vector $\widetilde{\boldsymbol{\beta}} \in \mathbb{R}_{+}^{d}$ and the matrix $\overline{\boldsymbol{C}} \in \mathbb{R}_{+}^{d \times d}$, respectively, which appear in the drift and diffusion coefficients of the SDE (4.3), respectively.

5.1 Example. Let $\left(\boldsymbol{X}_{t}\right)_{t \in \mathbb{R}_{+}}$be a 2-type (2-dimensional) CBI process with parameters $(2, \boldsymbol{c}, \boldsymbol{\beta}, \boldsymbol{B}, \nu, \boldsymbol{\mu})$ such that

$$
\widetilde{\boldsymbol{B}}=\gamma\left[\begin{array}{cc}
-1 & 1 \\
1 & -1
\end{array}\right]
$$

with some $\gamma \in \mathbb{R}_{++}$. Let us suppose that $\mathbb{E}\left(\left\|\boldsymbol{X}_{0}\right\|\right)<\infty$ and the moment condition (2.3) holds. Then $\widetilde{\boldsymbol{B}}$ is irreducible, and the eigenvalues of $\widetilde{\boldsymbol{B}}$ are 0 and $-2 \gamma$ thus $s(\widetilde{\boldsymbol{B}})=0$, and hence $\left(\boldsymbol{X}_{t}\right)_{t \in \mathbb{R}_{+}}$is irreducible and critical. Further, we have

$$
\boldsymbol{u}=\boldsymbol{u}_{\widetilde{\boldsymbol{B}}}=\frac{1}{2}\left[\begin{array}{l}
1 \\
1
\end{array}\right], \quad \boldsymbol{v}=\boldsymbol{v}_{\widetilde{\boldsymbol{B}}}=\left[\begin{array}{l}
1 \\
1
\end{array}\right], \quad \overline{\boldsymbol{C}}=\frac{1}{2}\left(\boldsymbol{C}_{1}+\boldsymbol{C}_{2}\right) .
$$

5.2 Example. Let $\left(\boldsymbol{X}_{t}\right)_{t \in \mathbb{R}_{+}}$be an irreducible and critical $d$-type ( $d$-dimensional) CBI process with parameters $(d, \mathbf{0}, \mathbf{0}, \mathbf{0}, \nu, \boldsymbol{\mu})$. Let us suppose that the moment condition (2.3) holds. Then

$$
\begin{gathered}
\widetilde{\boldsymbol{B}}=\left(\widetilde{b}_{i, j}\right)_{i, j \in\{1, \ldots, d\}} \quad \text { with } \quad \widetilde{b}_{i, j}=\int_{U_{d}}\left(z_{i}-\delta_{i, j}\right)^{+} \mu_{j}(\mathrm{~d} \boldsymbol{z}), \\
\widetilde{\boldsymbol{\beta}}=\int_{U_{d}} \boldsymbol{z} \nu(\mathrm{d} \boldsymbol{z}), \quad \boldsymbol{C}_{k}=\int_{U_{d}} \boldsymbol{z} \boldsymbol{z}^{\top} \mu_{k}(\mathrm{~d} \boldsymbol{z}), \quad k \in\{1, \ldots, d\},
\end{gathered}
$$

and $\bar{C}$ is given by (4.4) with the given $\boldsymbol{C}_{k}, k \in\{1, \ldots, d\}$.

5.3 Example. Let $\left(\boldsymbol{X}_{t}\right)_{t \in \mathbb{R}_{+}}$be an irreducible and critical $d$-type ( $d$-dimensional) CBI process with parameters $(d, \boldsymbol{c}, \boldsymbol{\beta}, \boldsymbol{B}, 0, \mathbf{0})$. Then

$$
\widetilde{\boldsymbol{B}}=\boldsymbol{B}, \quad \widetilde{\boldsymbol{\beta}}=\boldsymbol{\beta}, \quad \boldsymbol{C}_{k}=2 c_{k} \boldsymbol{e}_{k} \boldsymbol{e}_{k}^{\top}, \quad k \in\{1, \ldots, d\}, \quad \overline{\boldsymbol{C}}=2 \sum_{k=1}^{d} c_{k}\left(\boldsymbol{e}_{k}^{\top} \boldsymbol{u}\right)\left(\boldsymbol{e}_{k} \boldsymbol{e}_{k}^{\top}\right) .
$$

\section{Proof of Theorem 4.1}

The technique of the proof is somewhat similar to that of Theorem 3.1 in Ispány and Pap [17]. Let us introduce the notations

$$
\begin{gathered}
\widetilde{\widetilde{\boldsymbol{\beta}}}:=\left(\int_{0}^{1} \mathrm{e}^{s \widetilde{\boldsymbol{B}}} \mathrm{d} s\right) \widetilde{\boldsymbol{\beta}} \in \mathbb{R}_{+}^{d}, \\
\widetilde{\boldsymbol{C}}:=\sum_{k=1}^{d} \int_{0}^{1}\left(\boldsymbol{e}_{k}^{\top} \mathrm{e}^{(1-s) \widetilde{\boldsymbol{B}}} \boldsymbol{u}\right) \mathrm{e}^{s \widetilde{\boldsymbol{B}}} \boldsymbol{C}_{k} \mathrm{e}^{s \widetilde{\boldsymbol{B}}^{\top}} \mathrm{d} s \in \mathbb{R}_{+}^{d \times d} .
\end{gathered}
$$


Note that $\widetilde{\widetilde{\beta}}=\mathbb{E}\left(\boldsymbol{X}_{1}\right)$ if $\boldsymbol{X}_{0}=\mathbf{0}$, see (2.4).

The process $\left(\boldsymbol{X}_{t}\right)_{t \in \mathbb{R}_{+}}$is a time-homogeneous Markov process, hence Lemma 3.4 in Barczy et al. 4] implies

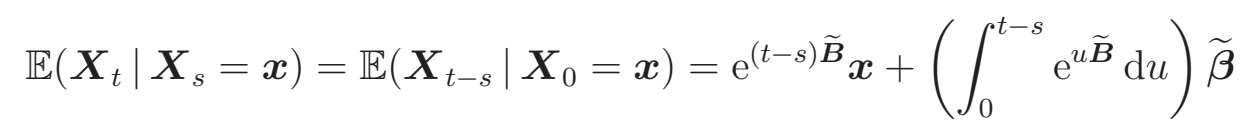

for all $\boldsymbol{x} \in \mathbb{R}_{+}^{d}$ and $s, t \in \mathbb{R}_{+}$with $s<t$. Using this formula, in order to prove (4.2), let us introduce the sequence

$$
\begin{aligned}
\boldsymbol{M}_{k} & :=\boldsymbol{X}_{k}-\mathbb{E}\left(\boldsymbol{X}_{k} \mid \mathcal{F}_{k-1}^{\boldsymbol{X}}\right)=\boldsymbol{X}_{k}-\mathbb{E}\left(\boldsymbol{X}_{k} \mid \boldsymbol{X}_{k-1}\right) \\
& =\boldsymbol{X}_{k}-\mathrm{e}^{\widetilde{\boldsymbol{B}}} \boldsymbol{X}_{k-1}-\left(\int_{0}^{1} \mathrm{e}^{u \widetilde{\boldsymbol{B}}} \mathrm{d} u\right) \widetilde{\boldsymbol{\beta}}=\boldsymbol{X}_{k}-\mathrm{e}^{\widetilde{\boldsymbol{B}}} \boldsymbol{X}_{k-1}-\widetilde{\widetilde{\beta}}, \quad k \in \mathbb{N},
\end{aligned}
$$

which is a sequence of martingale differences with respect to the filtration $\left(\mathcal{F}_{k}^{\boldsymbol{X}}\right)_{k \in \mathbb{Z}_{+}}$, where, for each $t \in \mathbb{R}_{+}, \mathcal{F}_{t}^{\boldsymbol{X}}$ denotes the $\sigma$-algebra generated by $\left(\boldsymbol{X}_{s}\right)_{s \in[0, t]}$. Consider the random step processes

$$
\mathcal{M}_{t}^{(n)}:=n^{-1}\left(\boldsymbol{X}_{0}+\sum_{k=1}^{\lfloor n t\rfloor} \boldsymbol{M}_{k}\right), \quad t \in \mathbb{R}_{+}, \quad n \in \mathbb{N} .
$$

First we will verify convergence

$$
\mathcal{M}^{(n)} \stackrel{\mathcal{D}}{\longrightarrow} \mathcal{M} \quad \text { as } n \rightarrow \infty,
$$

where $\left(\mathcal{M}_{t}\right)_{t \in \mathbb{R}_{+}}$is the unique strong solution of the SDE

$$
\mathrm{d} \mathcal{M}_{t}=\sqrt{\left\langle\boldsymbol{v}, \mathcal{M}_{t}+t \widetilde{\boldsymbol{\beta}}\right\rangle^{+} \widetilde{\boldsymbol{C}}} \mathrm{d} \mathcal{W}_{t}, \quad t \in \mathbb{R}_{+}, \quad \mathcal{M}_{0}=\mathbf{0}
$$

where $\left(\mathcal{W}_{t}\right)_{t \in \mathbb{R}_{+}}$is a $d$-dimensional standard Brownian motion. Here we note that the matrix $\left\langle\boldsymbol{v}, \mathcal{M}_{t}+t \widetilde{\widetilde{\beta}}\right\rangle^{+} \widetilde{\boldsymbol{C}}$ is symmetric and positive semidefinite, and $\sqrt{\left\langle\boldsymbol{v}, \mathcal{M}_{t}+t \widetilde{\widetilde{\beta}}\right\rangle^{+} \widetilde{\boldsymbol{C}}}$ denotes its unique symmetric and positive semidefinite square root. We want to apply Theorem C.1 with $\mathcal{U}:=\mathcal{M}, \boldsymbol{U}_{k}^{(n)}:=n^{-1} \boldsymbol{M}_{k}, n, k \in \mathbb{N}, \boldsymbol{U}_{0}^{(n)}:=n^{-1} \boldsymbol{X}_{0}, n \in \mathbb{N}, \mathcal{F}_{k}^{(n)}:=\mathcal{F}_{k}^{\boldsymbol{X}}$ for $n \in \mathbb{N}$, $k \in \mathbb{Z}_{+}$, and with the coefficient function $\gamma: \mathbb{R}_{+} \times \mathbb{R}^{d} \rightarrow \mathbb{R}^{d \times d}$ of the SDE (6.4) given by

$$
\gamma(t, \boldsymbol{x}):=\sqrt{\langle\boldsymbol{v}, \boldsymbol{x}+t \widetilde{\widetilde{\boldsymbol{\beta}}}\rangle^{+} \widetilde{\boldsymbol{C}}}, \quad(t, \boldsymbol{x}) \in \mathbb{R}_{+} \times \mathbb{R}^{d}
$$

The aim of the following discussion is to show that the SDE (6.4) has a pathwise unique strong solution $\left(\mathcal{M}_{t}^{\left(\boldsymbol{y}_{0}\right)}\right)_{t \in \mathbb{R}_{+}}$with initial value $\mathcal{M}_{0}^{\left(\boldsymbol{y}_{0}\right)}=\boldsymbol{y}_{0}$ for all $\boldsymbol{y}_{0} \in \mathbb{R}^{d}$. First observe that if $\left(\mathcal{M}_{t}^{\left(\boldsymbol{y}_{0}\right)}\right)_{t \in \mathbb{R}_{+}}$is a strong solution of the $\operatorname{SDE}(\underline{6.4})$ with initial value $\boldsymbol{\mathcal { M }}_{0}^{\left(\boldsymbol{y}_{0}\right)}=\boldsymbol{y}_{0}$, then, by Itô's formula, the process $\left(\mathcal{P}_{t}, \mathcal{Q}_{t}\right)_{t \in \mathbb{R}_{+}}$, defined by

$$
\mathcal{P}_{t}:=\left\langle\boldsymbol{v}, \mathcal{M}_{t}^{\left(\boldsymbol{y}_{0}\right)}+t \widetilde{\widetilde{\beta}}\right\rangle, \quad \mathcal{Q}_{t}:=\mathcal{M}_{t}^{\left(\boldsymbol{y}_{0}\right)}-\mathcal{P}_{t} \boldsymbol{u}, \quad t \in \mathbb{R}_{+},
$$


is a strong solution of the SDE

$$
\left\{\begin{array}{l}
\mathrm{d} \mathcal{P}_{t}=\boldsymbol{v}^{\top} \widetilde{\widetilde{\beta}} \mathrm{d} t+\sqrt{\mathcal{P}_{t}^{+}} \boldsymbol{v}^{\top} \sqrt{\widetilde{\boldsymbol{C}}} \mathrm{d} \mathcal{W}_{t}, \\
\mathrm{~d} \mathcal{Q}_{t}=-\boldsymbol{\Pi} \widetilde{\widetilde{\beta}} \mathrm{d} t+\sqrt{\mathcal{P}_{t}^{+}}\left(\boldsymbol{I}_{d}-\boldsymbol{\Pi}\right) \sqrt{\widetilde{\boldsymbol{C}}} \mathrm{d} \mathcal{W}_{t}
\end{array} \quad t \in \mathbb{R}_{+},\right.
$$

with initial value $\left(\mathcal{P}_{0}, \mathcal{Q}_{0}\right)=\left(\boldsymbol{v}^{\top} \boldsymbol{y}_{0},\left(\boldsymbol{I}_{d}-\boldsymbol{\Pi}\right) \boldsymbol{y}_{0}\right)$, where $\boldsymbol{\Pi}:=\boldsymbol{\Pi}_{\widetilde{\boldsymbol{B}}}$, see (iii) of Lemma A.3. Indeed, the first SDE of (6.5) is an easy consequence of the SDE (6.4). The second one can be checked as follows. By Itô's formula,

$$
\begin{aligned}
\mathrm{d} \mathcal{Q}_{t} & =\mathrm{d} \mathcal{M}_{t}^{\left(\boldsymbol{y}_{0}\right)}-\boldsymbol{u} \mathrm{d} \mathcal{P}_{t}=\mathrm{d} \mathcal{M}_{t}^{\left(\boldsymbol{y}_{0}\right)}-\boldsymbol{u} \boldsymbol{v}^{\top}\left(\mathrm{d} \mathcal{M}_{t}^{\left(\boldsymbol{y}_{0}\right)}+\widetilde{\widetilde{\boldsymbol{\beta}}} \mathrm{d} t\right)=-\boldsymbol{\Pi} \widetilde{\widetilde{\boldsymbol{\beta}}} \mathrm{d} t+\left(\boldsymbol{I}_{d}-\boldsymbol{\Pi}\right) \mathrm{d} \mathcal{M}_{t}^{\left(\boldsymbol{y}_{0}\right)} \\
& =-\boldsymbol{\Pi} \widetilde{\widetilde{\boldsymbol{\beta}}} \mathrm{d} t+\left(\boldsymbol{I}_{d}-\boldsymbol{\Pi}\right) \sqrt{\left\langle\boldsymbol{v}, \mathcal{M}_{t}^{\left(\boldsymbol{y}_{0}\right)}+t \widetilde{\boldsymbol{\beta}}\right\rangle^{+} \widetilde{\boldsymbol{C}}} \mathrm{d} \mathcal{W}_{t} \\
& =-\boldsymbol{\Pi} \widetilde{\widetilde{\boldsymbol{\beta}}} \mathrm{d} t+\sqrt{\mathcal{P}_{t}^{+}}\left(\boldsymbol{I}_{d}-\boldsymbol{\Pi}\right) \sqrt{\widetilde{\boldsymbol{C}}} \mathrm{d} \mathcal{W}_{t}, \quad t \in \mathbb{R}_{+},
\end{aligned}
$$

where $\mathcal{Q}_{0}=\boldsymbol{y}_{0}-\left(\boldsymbol{v}^{\top} \boldsymbol{y}_{0}\right) \boldsymbol{u}=\boldsymbol{y}_{0}-\boldsymbol{u} \boldsymbol{v}^{\top} \boldsymbol{y}_{0}=\left(\boldsymbol{I}_{d}-\boldsymbol{\Pi}\right) \boldsymbol{y}_{0}$. Conversely, if $\left(\mathcal{P}_{t}^{\left(p_{0}, \boldsymbol{q}_{0}\right)}, \mathcal{Q}_{t}^{\left(p_{0}, \boldsymbol{q}_{0}\right)}\right)_{t \in \mathbb{R}_{+}}$ is a strong solution of the $\operatorname{SDE}(6.5)$ with initial value $\left(\mathcal{P}_{0}^{\left(p_{0}, \boldsymbol{q}_{0}\right)}, \boldsymbol{\mathcal { Q }}_{0}^{\left(p_{0}, \boldsymbol{q}_{0}\right)}\right)=\left(p_{0}, \boldsymbol{q}_{0}\right) \in \mathbb{R} \times \mathbb{R}^{d}$, then, again by Itô's formula,

$$
\mathcal{M}_{t}:=\mathcal{P}_{t}^{\left(p_{0}, \boldsymbol{q}_{0}\right)} \boldsymbol{u}+\mathcal{Q}_{t}^{\left(p_{0}, \boldsymbol{q}_{0}\right)}, \quad t \in \mathbb{R}_{+},
$$

is a strong solution of the SDE (6.4) with initial value $\boldsymbol{M}_{0}=p_{0} \boldsymbol{u}+\boldsymbol{q}_{0}$. The correspondence $\boldsymbol{y}_{0} \leftrightarrow\left(p_{0}, \boldsymbol{q}_{0}\right):=\left(\boldsymbol{v}^{\top} \boldsymbol{y}_{0},\left(\boldsymbol{I}_{d}-\boldsymbol{\Pi}\right) \boldsymbol{y}_{0}\right)$ is a bijection between $\mathbb{R}^{d}$ and $\mathbb{R} \times\left\{\boldsymbol{q} \in \mathbb{R}^{d}: \boldsymbol{v}^{\top} \boldsymbol{q}=0\right\}$, since $\boldsymbol{y}_{0}=p_{0} \boldsymbol{u}+\boldsymbol{q}_{0}$, and for all $\left(p_{0}, \boldsymbol{q}_{0}\right) \in \mathbb{R} \times\left\{\boldsymbol{q} \in \mathbb{R}^{d}: \boldsymbol{v}^{\top} \boldsymbol{q}=0\right\}$, by (iii) of Lemma A.3, $\boldsymbol{v}^{\top}\left(p_{0} \boldsymbol{u}+\boldsymbol{q}_{0}\right)=p_{0}$, and

$$
\left(\boldsymbol{I}_{d}-\boldsymbol{\Pi}\right)\left(p_{0} \boldsymbol{u}+\boldsymbol{q}_{0}\right)=p_{0} \boldsymbol{u}+\boldsymbol{q}_{0}-p_{0} \boldsymbol{\Pi} \boldsymbol{u}-\boldsymbol{\Pi} \boldsymbol{q}_{0}=p_{0} \boldsymbol{u}+\boldsymbol{q}_{0}-p_{0} \boldsymbol{u v} \boldsymbol{v}^{\top} \boldsymbol{u}-\boldsymbol{u v} \boldsymbol{v}^{\top} \boldsymbol{q}_{0}=\boldsymbol{q}_{0} .
$$

Hence it is enough to show that the SDE (6.5) has a pathwise unique strong solution $\left(\mathcal{P}_{t}^{\left(p_{0}, \boldsymbol{q}_{0}\right)}, \mathcal{Q}_{t}^{\left(p_{0}, \boldsymbol{q}_{0}\right)}\right)_{t \in \mathbb{R}_{+}}$with initial value $\left(\mathcal{P}_{0}^{\left(p_{0}, \boldsymbol{q}_{0}\right)}, \mathcal{Q}_{0}^{\left(p_{0}, \boldsymbol{q}_{0}\right)}\right)=\left(p_{0}, \boldsymbol{q}_{0}\right)$ for all $\left(p_{0}, \boldsymbol{q}_{0}\right) \in$ $\mathbb{R} \times\left\{\boldsymbol{q} \in \mathbb{R}^{d}: \boldsymbol{v}^{\top} \boldsymbol{q}=0\right\} \quad$ (actually, it turns out that it has a pathwise unique strong solution in case of any $\left.\left(p_{0}, \boldsymbol{q}_{0}\right) \in \mathbb{R} \times \mathbb{R}^{d}\right)$. The first equation of (6.5) can be written in the form

$$
\mathrm{d} \mathcal{P}_{t}=\boldsymbol{v}^{\top} \widetilde{\widetilde{\boldsymbol{\beta}}} \mathrm{d} t+\sqrt{\mathcal{P}_{t}^{+}} \sqrt{\langle\widetilde{\boldsymbol{C}} \boldsymbol{v}, \boldsymbol{v}\rangle} \mathrm{d} \mathcal{W}_{t}, \quad t \in \mathbb{R}_{+},
$$

where $\left(\mathcal{W}_{t}\right)_{t \in \mathbb{R}_{+}}$is a 1-dimensional standard Brownian motion. Indeed, $\langle\widetilde{\boldsymbol{C}} \boldsymbol{v}, \boldsymbol{v}\rangle=$ $\left(\boldsymbol{v}^{\top} \sqrt{\widetilde{\boldsymbol{C}}}\right)\left(\boldsymbol{v}^{\top} \sqrt{\widetilde{\boldsymbol{C}}}\right)^{\top}=\left\|\boldsymbol{v}^{\top} \sqrt{\widetilde{\boldsymbol{C}}}\right\|^{2}$, and hence if $\boldsymbol{v}^{\top} \sqrt{\widetilde{\boldsymbol{C}}}=\mathbf{0} \in \mathbb{R}^{1 \times d}$, then the above mentioned rewriting of the SDE (6.5) is trivial, and if $\boldsymbol{v}^{\top} \sqrt{\widetilde{\boldsymbol{C}}} \neq \mathbf{0} \in \mathbb{R}^{1 \times d}$, then $\mathcal{W}_{t}:=$ $\langle\widetilde{\boldsymbol{C}} \boldsymbol{v}, \boldsymbol{v}\rangle^{-1 / 2} \boldsymbol{v}^{\top} \sqrt{\widetilde{\boldsymbol{C}}} \mathcal{W}_{t}, t \in \mathbb{R}_{+}$, is a 1-dimensional standard Brownian motion. Hence, by Remark 4.4, the first equation of the SDE (6.5) has a pathwise unique strong solution $\left(\mathcal{P}_{t}^{\left(p_{0}\right)}\right)_{t \in \mathbb{R}_{+}}$ with initial value $\mathcal{P}_{0}^{\left(p_{0}\right)}=p_{0}$ for all $p_{0} \in \mathbb{R}$. Clearly, the second equation of the SDE (6.5) has a pathwise unique strong solution

$$
\mathcal{Q}_{t}^{\left(p_{0}, \boldsymbol{q}_{0}\right)}=\boldsymbol{q}_{0}-\boldsymbol{\Pi} \widetilde{\widetilde{\boldsymbol{\beta}}} t+\left(\boldsymbol{I}_{d}-\boldsymbol{\Pi}\right) \sqrt{\widetilde{\boldsymbol{C}}} \int_{0}^{t} \sqrt{\left(\mathcal{P}_{s}^{\left(p_{0}\right)}\right)+} \mathrm{d} \mathcal{W}_{s}, \quad t \in \mathbb{R}_{+},
$$


with initial value $\mathcal{Q}_{0}^{\left(p_{0}, \boldsymbol{q}_{0}\right)}=\boldsymbol{q}_{0}$ for all $\left(p_{0}, \boldsymbol{q}_{0}\right) \in \mathbb{R} \times \mathbb{R}^{d}$. Consequently, the SDE (6.5), and hence the SDE (6.4) admit a pathwise unique strong solution with an arbitrary initial value.

Now we show that conditions (i) and (ii) of Theorem C.1 hold. We have to check that for each $T>0$,

$$
\begin{aligned}
& \sup _{t \in[0, T]}\left\|\frac{1}{n^{2}} \sum_{k=1}^{\lfloor n t\rfloor} \operatorname{Var}\left(\boldsymbol{M}_{k} \mid \mathcal{F}_{k-1}^{\boldsymbol{X}}\right)-\left(\int_{0}^{t}\left(\mathcal{R}_{s}^{(n)}\right)^{+} \mathrm{d} s\right) \widetilde{\boldsymbol{C}}\right\| \stackrel{\mathbb{P}}{\longrightarrow} 0, \\
& \frac{1}{n^{2}} \sum_{k=1}^{\lfloor n T\rfloor} \mathbb{E}\left(\left\|\boldsymbol{M}_{k}\right\|^{2} \mathbb{1}_{\left\{\left\|\boldsymbol{M}_{k}\right\|>n \theta\right\}} \mid \mathcal{F}_{k-1}^{\boldsymbol{X}}\right) \stackrel{\mathbb{P}}{\longrightarrow} 0 \quad \text { for all } \theta>0
\end{aligned}
$$

as $n \rightarrow \infty$, where the process $\left(\mathcal{R}_{s}^{(n)}\right)_{s \in \mathbb{R}_{+}}$is defined by

$$
\mathcal{R}_{s}^{(n)}:=\boldsymbol{v}^{\top}\left(\mathcal{M}_{s}^{(n)}+s \widetilde{\widetilde{\beta}}\right), \quad s \in \mathbb{R}_{+}, \quad n \in \mathbb{N} .
$$

By (6.1),

$$
\begin{aligned}
\boldsymbol{\mathcal { R }}_{s}^{(n)} & =\boldsymbol{v}^{\top}\left(n^{-1} \boldsymbol{X}_{0}+n^{-1} \sum_{k=1}^{\lfloor n s\rfloor}\left(\boldsymbol{X}_{k}-\mathrm{e}^{\widetilde{\boldsymbol{B}}} \boldsymbol{X}_{k-1}-\widetilde{\widetilde{\boldsymbol{\beta}}}\right)+s \widetilde{\widetilde{\beta}}\right) \\
& =n^{-1} \boldsymbol{v}^{\top} \boldsymbol{X}_{0}+n^{-1} \sum_{k=1}^{\lfloor n s\rfloor}\left(\boldsymbol{v}^{\top} \boldsymbol{X}_{k}-\boldsymbol{v}^{\top} \mathrm{e}^{\widetilde{\boldsymbol{B}}} \boldsymbol{X}_{k-1}-\boldsymbol{v}^{\top} \widetilde{\boldsymbol{\beta}}\right)+s \boldsymbol{v}^{\top} \widetilde{\widetilde{\beta}} \\
& =n^{-1} \boldsymbol{v}^{\top} \boldsymbol{X}_{0}+n^{-1} \sum_{k=1}^{\lfloor n s\rfloor}\left(\boldsymbol{v}^{\top} \boldsymbol{X}_{k}-\boldsymbol{v}^{\top} \boldsymbol{X}_{k-1}-\boldsymbol{v}^{\top} \widetilde{\boldsymbol{\beta}}\right)+s \boldsymbol{v}^{\top} \widetilde{\widetilde{\beta}} \\
& =n^{-1} \boldsymbol{v}^{\top} \boldsymbol{X}_{\lfloor n s\rfloor}+\left(s-\frac{\lfloor n s\rfloor}{n}\right) \boldsymbol{v}^{\top} \widetilde{\widetilde{\beta}}
\end{aligned}
$$

where we used that $\boldsymbol{v}$ is a left eigenvector of $\mathrm{e}^{\widetilde{\boldsymbol{B}}}$ belonging to the eigenvalue 1 (due to the fact that $\boldsymbol{v}$ is a left eigenvector of $\widetilde{\boldsymbol{B}}$ corresponding to the eigenvalue $s(\widetilde{\boldsymbol{B}})=0)$. Thus $\left(\boldsymbol{\mathcal { R }}_{s}^{(n)}\right)^{+}=\boldsymbol{\mathcal { R }}_{s}^{(n)}$, and

$$
\begin{aligned}
\int_{0}^{t}\left(\boldsymbol{\mathcal { R }}_{s}^{(n)}\right)^{+} \mathrm{d} s= & \frac{1}{n^{2}} \sum_{\ell=0}^{\lfloor n t\rfloor-1} \boldsymbol{v}^{\top} \boldsymbol{X}_{\ell}+\left(t-\frac{\lfloor n t\rfloor}{n}\right) \frac{1}{n} \boldsymbol{v}^{\top} \boldsymbol{X}_{\lfloor n t\rfloor} \\
& +\frac{t^{2}}{2} \boldsymbol{v}^{\top} \widetilde{\boldsymbol{\beta}}-\frac{1}{n^{2}}\left(\sum_{\ell=1}^{\lfloor n t\rfloor-1} \ell\right) \boldsymbol{v}^{\top} \widetilde{\boldsymbol{\beta}}-\left(t-\frac{\lfloor n t\rfloor}{n}\right) \frac{\lfloor n t\rfloor}{n} \boldsymbol{v}^{\top} \widetilde{\widetilde{\beta}} \\
= & \frac{1}{n^{2}} \sum_{\ell=0}^{\lfloor n t\rfloor-1} \boldsymbol{v}^{\top} \boldsymbol{X}_{\ell}+\frac{n t-\lfloor n t\rfloor}{n^{2}} \boldsymbol{v}^{\top} \boldsymbol{X}_{\lfloor n t\rfloor}+\frac{\lfloor n t\rfloor+(n t-\lfloor n t\rfloor)^{2}}{2 n^{2}} \boldsymbol{v}^{\top} \widetilde{\boldsymbol{\beta}} .
\end{aligned}
$$

We have $\operatorname{Var}\left(\boldsymbol{M}_{k} \mid \mathcal{F}_{k-1}^{\boldsymbol{X}}\right)=\operatorname{Var}\left(\boldsymbol{X}_{k} \mid \boldsymbol{X}_{k-1}\right)$ and $\operatorname{Var}\left(\boldsymbol{X}_{k} \mid \boldsymbol{X}_{k-1}=\boldsymbol{x}\right)=\operatorname{Var}\left(\boldsymbol{X}_{1} \mid \boldsymbol{X}_{0}=\boldsymbol{x}\right)$ for all $\boldsymbol{x} \in \mathbb{R}_{+}^{d}$, since $\left(\boldsymbol{X}_{t}\right)_{t \in \mathbb{R}_{+}}$is a time-homogeneous Markov process. Hence Lemma 4.4 in 
Barczy et al. [5] implies

$$
\frac{1}{n^{2}} \sum_{k=1}^{\lfloor n t\rfloor} \operatorname{Var}\left(\boldsymbol{M}_{k} \mid \mathcal{F}_{k-1}^{\boldsymbol{X}}\right)=\frac{\lfloor n t\rfloor}{n^{2}} \boldsymbol{V}+\frac{1}{n^{2}} \sum_{k=1}^{\lfloor n t\rfloor} \sum_{\ell=1}^{d} \int_{0}^{1}\left(\boldsymbol{e}_{\ell}^{\top} \mathrm{e}^{(1-s) \widetilde{\boldsymbol{B}}} \boldsymbol{X}_{k-1}\right) \mathrm{e}^{s \widetilde{\boldsymbol{B}}} \boldsymbol{C}_{\ell} \mathrm{e}^{s \widetilde{\boldsymbol{B}}^{\top}} \mathrm{d} s,
$$

where

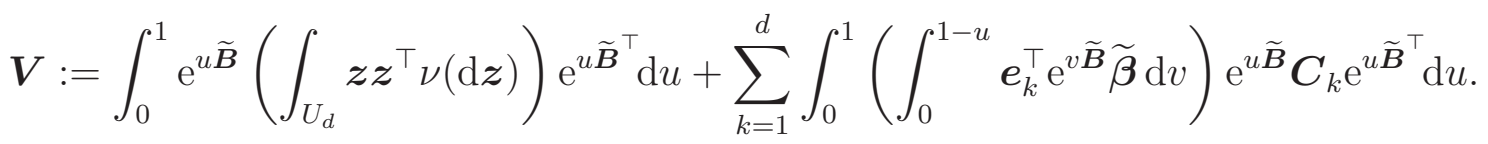

Hence, in order to show (6.7), it suffices to prove

$$
\begin{aligned}
& n^{-2} \sup _{t \in[0, T]} \sum_{k=0}^{\lfloor n t\rfloor-1}\left\|\sum_{\ell=1}^{d} \int_{0}^{1}\left(\boldsymbol{e}_{\ell}^{\top} \mathrm{e}^{(1-s) \widetilde{\boldsymbol{B}}} \boldsymbol{X}_{k}\right) \mathrm{e}^{s \widetilde{\boldsymbol{B}}} \boldsymbol{C}_{\ell} \mathrm{e}^{s \widetilde{\boldsymbol{B}}^{\top}} \mathrm{d} s-\left(\boldsymbol{v}^{\top} \boldsymbol{X}_{k}\right) \widetilde{\boldsymbol{C}}\right\| \stackrel{\mathbb{P}}{\longrightarrow} 0, \\
& n^{-2} \sup _{t \in[0, T]}\left\|\boldsymbol{X}_{\lfloor n t\rfloor}\right\| \stackrel{\mathbb{P}}{\longrightarrow} 0
\end{aligned}
$$

as $n \rightarrow \infty$. Observe that

$$
\begin{aligned}
& \left(\boldsymbol{v}^{\top} \boldsymbol{X}_{k}\right) \widetilde{\boldsymbol{C}}=\sum_{\ell=1}^{d} \int_{0}^{1}\left(\boldsymbol{e}_{\ell}^{\top} \mathrm{e}^{(1-s) \widetilde{\boldsymbol{B}}} \boldsymbol{u}\right)\left(\boldsymbol{v}^{\top} \boldsymbol{X}_{k}\right) \mathrm{e}^{s \widetilde{\boldsymbol{B}}} \boldsymbol{C}_{\ell} \mathrm{e}^{s \widetilde{\boldsymbol{B}}^{\top}} \mathrm{d} s \\
& =\sum_{\ell=1}^{d} \int_{0}^{1}\left(\boldsymbol{e}_{\ell}^{\top} \mathrm{e}^{(1-s) \widetilde{\boldsymbol{B}}} \boldsymbol{\Pi} \boldsymbol{X}_{k}\right) \mathrm{e}^{s \widetilde{\boldsymbol{B}}} \boldsymbol{C}_{\ell} \mathrm{e}^{s \widetilde{\boldsymbol{B}}^{\top}} \mathrm{d} s, \quad k \in \mathbb{Z}_{+} .
\end{aligned}
$$

From (6.1) we obtain the recursion

$$
\boldsymbol{X}_{k}=\mathrm{e}^{\widetilde{\boldsymbol{B}}} \boldsymbol{X}_{k-1}+\boldsymbol{M}_{k}+\widetilde{\widetilde{\boldsymbol{\beta}}}, \quad k \in \mathbb{N},
$$

hence we conclude

$$
\boldsymbol{X}_{k}=\mathrm{e}^{k \widetilde{\boldsymbol{B}}} \boldsymbol{X}_{0}+\sum_{j=1}^{k} \mathrm{e}^{(k-j) \widetilde{\boldsymbol{B}}}\left(\boldsymbol{M}_{j}+\widetilde{\widetilde{\boldsymbol{\beta}}}\right), \quad k \in \mathbb{N} .
$$

Using (6.11), for all $k \in \mathbb{Z}_{+}$, we obtain

$$
\begin{aligned}
& \sum_{\ell=1}^{d} \int_{0}^{1}\left(\boldsymbol{e}_{\ell}^{\top} \mathrm{e}^{(1-s) \widetilde{\boldsymbol{B}}} \boldsymbol{X}_{k}\right) \mathrm{e}^{s \widetilde{\boldsymbol{B}}} \boldsymbol{C}_{\ell} \mathrm{e}^{s \widetilde{\boldsymbol{B}}^{\top}} \mathrm{d} s-\left(\boldsymbol{v}^{\top} \boldsymbol{X}_{k}\right) \widetilde{\boldsymbol{C}} \\
& \quad=\sum_{\ell=1}^{d} \int_{0}^{1}\left[\boldsymbol{e}_{\ell}^{\top} \mathrm{e}^{(1-s) \widetilde{\boldsymbol{B}}}\left(\boldsymbol{I}_{d}-\boldsymbol{\Pi}\right) \boldsymbol{X}_{k}\right] \mathrm{e}^{s \widetilde{\boldsymbol{B}}} \boldsymbol{C}_{\ell} \mathrm{e}^{s \widetilde{\boldsymbol{B}}} \mathrm{d} s \\
& \quad=\sum_{\ell=1}^{d} \int_{0}^{1}\left[\boldsymbol{e}_{\ell}^{\top} \mathrm{e}^{(1-s) \widetilde{\boldsymbol{B}}}\left(\boldsymbol{I}_{d}-\boldsymbol{\Pi}\right)\left\{\mathrm{e}^{k \widetilde{\boldsymbol{B}}} \boldsymbol{X}_{0}+\sum_{j=1}^{k} \mathrm{e}^{(k-j) \widetilde{\boldsymbol{B}}}\left(\boldsymbol{M}_{j}+\widetilde{\boldsymbol{\beta}}\right)\right\}\right] \mathrm{e}^{s \widetilde{\boldsymbol{B}}} \boldsymbol{C}_{\ell} \mathrm{e}^{s \widetilde{\boldsymbol{B}}^{\top} \mathrm{d} s} \\
& \quad=\sum_{\ell=1}^{d} \int_{0}^{1}\left[\boldsymbol{e}_{\ell}^{\top} \mathrm{e}^{(1-s) \widetilde{\boldsymbol{B}}}\left\{\left(\mathrm{e}^{k \widetilde{\boldsymbol{B}}}-\boldsymbol{\Pi}\right) \boldsymbol{X}_{0}+\sum_{j=1}^{k}\left(\mathrm{e}^{(k-j) \widetilde{\boldsymbol{B}}}-\boldsymbol{\Pi}\right)\left(\boldsymbol{M}_{j}+\widetilde{\boldsymbol{\beta}}\right)\right\}\right] \mathrm{e}^{s \widetilde{\boldsymbol{B}}} \boldsymbol{C}_{\ell} \mathrm{e}^{s \widetilde{\boldsymbol{B}}} \mathrm{d} s,
\end{aligned}
$$


since, by (iii) of Lemma $\underline{\text { A.3 }}, \quad \Pi \mathrm{e}^{\widetilde{\boldsymbol{B}}}=\left(\lim _{t \rightarrow \infty} \mathrm{e}^{t \widetilde{\boldsymbol{B}}}\right) \mathrm{e}^{\widetilde{\boldsymbol{B}}}=\lim _{t \rightarrow \infty} \mathrm{e}^{(t+1) \widetilde{\boldsymbol{B}}}=\boldsymbol{\Pi}$ implies $\quad\left(\boldsymbol{I}_{d}-\right.$ $\boldsymbol{\Pi}) \mathrm{e}^{(k-j) \widetilde{\boldsymbol{B}}}=\mathrm{e}^{(k-j) \widetilde{\boldsymbol{B}}}-\boldsymbol{\Pi}$. Hence, by (iv) of Lemma A.3,

$$
\begin{aligned}
& \sum_{k=0}^{\lfloor n t\rfloor}\left\|\sum_{\ell=1}^{d} \int_{0}^{1}\left(\boldsymbol{e}_{\ell}^{\top} \mathrm{e}^{(1-s) \widetilde{\boldsymbol{B}}} \boldsymbol{X}_{k}\right) \mathrm{e}^{\widetilde{\boldsymbol{B}}} \boldsymbol{C}_{\ell} \mathrm{e}^{\widetilde{\boldsymbol{B}}^{\top}} \mathrm{d} s-\left(\boldsymbol{v}^{\top} \boldsymbol{X}_{k}\right) \widetilde{\boldsymbol{C}}\right\| \\
& \quad \leqslant c \sum_{k=0}^{\lfloor n t\rfloor} \sum_{\ell=1}^{d}\left\{\mathrm{e}^{-k \kappa}\left\|\boldsymbol{X}_{0}\right\|+\sum_{j=1}^{k} \mathrm{e}^{-(k-j) \kappa}\left\|\boldsymbol{M}_{j}+\widetilde{\widetilde{\boldsymbol{\beta}}}\right\|\right\} \int_{0}^{1}\left\|\mathrm{e}^{(1-s) \widetilde{\boldsymbol{B}}}\right\|\left\|\mathrm{e}^{s \widetilde{\boldsymbol{B}}} \boldsymbol{C}_{\ell} \mathrm{e}^{s \widetilde{\boldsymbol{B}}^{\top}}\right\| \mathrm{d} s \\
& \leqslant c(c+\|\boldsymbol{\Pi}\|)^{3} \sum_{\ell=1}^{d}\left\|\boldsymbol{C}_{\ell}\right\|\left\{\left\|\boldsymbol{X}_{0}\right\| \sum_{k=0}^{\lfloor n t\rfloor} \mathrm{e}^{-k \kappa}+\sum_{j=1}^{\lfloor n t\rfloor} \sum_{k=j}^{\lfloor n t\rfloor} \mathrm{e}^{-(k-j) \kappa}\left(\left\|\boldsymbol{M}_{j}\right\|+\|\widetilde{\widetilde{\boldsymbol{\beta}}}\|\right)\right\} \\
& \leqslant \frac{c(c+\|\boldsymbol{\Pi}\|)^{3}}{1-\mathrm{e}^{-\kappa}}\left(\left\|\boldsymbol{X}_{0}\right\|+\lfloor n t\rfloor \cdot\|\widetilde{\boldsymbol{\beta}}\|+\sum_{j=1}^{\lfloor n t\rfloor}\left\|\boldsymbol{M}_{j}\right\|\right) \sum_{\ell=1}^{d}\left\|\boldsymbol{C}_{\ell}\right\|,
\end{aligned}
$$

since $\sum_{k=0}^{\lfloor n t\rfloor} \mathrm{e}^{-k \kappa}<\sum_{k=0}^{\infty} \mathrm{e}^{-k \kappa}=\frac{1}{1-\mathrm{e}^{-\kappa}}$, and, by (iv) of Lemma A.3.

$$
\left\|\mathrm{e}^{t \widetilde{\boldsymbol{B}}^{\top}}\right\|=\left\|\mathrm{e}^{t \widetilde{\boldsymbol{B}}}\right\| \leqslant c+\|\boldsymbol{\Pi}\|, \quad t \in \mathbb{R}_{+},
$$

where $c:=c_{\widetilde{\boldsymbol{B}}} \in \mathbb{R}_{++}$and $\kappa:=\kappa_{\widetilde{\boldsymbol{B}}} \in \mathbb{R}_{++}$are given in (iv) of Lemma A.3.

Moreover, by (6.11) and (6.12),

$$
\begin{aligned}
\left\|\boldsymbol{X}_{\lfloor n t\rfloor}\right\| & \leqslant\left\|\mathrm{e}^{\lfloor n t\rfloor \widetilde{\boldsymbol{B}}}\right\|\left\|\boldsymbol{X}_{0}\right\|+\sum_{j=1}^{\lfloor n t\rfloor}\left\|\mathrm{e}^{(\lfloor n t\rfloor-j) \widetilde{\boldsymbol{B}}}\right\|\left\|\boldsymbol{M}_{j}+\widetilde{\widetilde{\boldsymbol{\beta}}}\right\| \\
& \leqslant(c+\|\boldsymbol{\Pi}\|)\left(\left\|\boldsymbol{X}_{0}\right\|+\lfloor n t\rfloor \cdot\|\widetilde{\widetilde{\boldsymbol{\beta}}}\|+\sum_{j=1}^{\lfloor n t\rfloor}\left\|\boldsymbol{M}_{j}\right\|\right) .
\end{aligned}
$$

Consequently, in order to prove (6.10), it suffices to show

$$
\frac{1}{n^{2}} \sum_{j=1}^{\lfloor n T\rfloor}\left\|\boldsymbol{M}_{j}\right\| \stackrel{\mathbb{P}}{\longrightarrow} 0 \quad \text { as } n \rightarrow \infty .
$$

In fact, Lemma B.3 yields $n^{-2} \sum_{j=1}^{\lfloor n T\rfloor} \mathbb{E}\left(\left\|\boldsymbol{M}_{j}\right\|\right) \leqslant n^{-2} \sum_{j=1}^{\lfloor n T\rfloor} \sqrt{\mathbb{E}\left(\left\|\boldsymbol{M}_{j}\right\|^{2}\right)} \rightarrow 0$ as $n \rightarrow \infty$, thus we obtain (6.7).

Next we check condition (6.8). We have

$$
\mathbb{E}\left(\left\|\boldsymbol{M}_{k}\right\|^{2} \mathbb{1}_{\left\{\left\|\boldsymbol{M}_{k}\right\|>n \theta\right\}} \mid \mathcal{F}_{k-1}^{\boldsymbol{X}}\right) \leqslant n^{-2} \theta^{-2} \mathbb{E}\left(\left\|\boldsymbol{M}_{k}\right\|^{4} \mid \mathcal{F}_{k-1}^{\boldsymbol{X}}\right) .
$$

Moreover, $n^{-4} \sum_{k=1}^{\lfloor n T\rfloor} \mathbb{E}\left(\left\|\boldsymbol{M}_{k}\right\|^{4}\right) \rightarrow 0$ as $n \rightarrow \infty$, since $\mathbb{E}\left(\left\|\boldsymbol{M}_{k}\right\|^{4}\right)=\mathrm{O}\left(k^{2}\right)$ by Lemma B.3 (at this point we used the moment condition $\mathbb{E}\left(\left\|\boldsymbol{X}_{0}\right\|^{4}\right)<\infty$ ). Thus we obtain (6.8), and hence, convergence (6.3). 
Applying a version of the continuous mapping theorem together with (6.3) and (6.11), we will show

$$
\left(\mathcal{X}_{t}^{(n)}\right)_{t \in \mathbb{R}_{+}} \stackrel{\mathcal{D}}{\longrightarrow}\left(\mathcal{X}_{t}\right)_{t \in \mathbb{R}_{+}} \quad \text { as } n \rightarrow \infty
$$

where

$$
\mathcal{X}_{t}:=\Pi\left(\mathcal{M}_{t}+t \widetilde{\widetilde{\beta}}\right), \quad t \in \mathbb{R}_{+},
$$

where $\left(\mathcal{M}_{t}\right)_{t \in \mathbb{R}_{+}}$is defined in (6.4). We want to apply Lemma D.1. By (6.11), $\boldsymbol{\mathcal { X }}^{(n)}=$ $\Psi_{n}\left(\mathcal{M}^{(n)}\right), n \in \mathbb{N}$, where the mapping $\Psi_{n}: \mathbb{D}\left(\mathbb{R}_{+}, \mathbb{R}^{d}\right) \rightarrow \mathbb{D}\left(\mathbb{R}_{+}, \mathbb{R}^{d}\right)$ is given by

$$
\Psi_{n}(f)(t):=\mathrm{e}^{\lfloor n t\rfloor \widetilde{\boldsymbol{B}}} f(0)+\sum_{j=1}^{\lfloor n t\rfloor} \mathrm{e}^{(\lfloor n t\rfloor-j) \widetilde{\boldsymbol{B}}}\left(f\left(\frac{j}{n}\right)-f\left(\frac{j-1}{n}\right)+n^{-1} \widetilde{\widetilde{\boldsymbol{\beta}}}\right)
$$

for $f \in \mathbb{D}\left(\mathbb{R}_{+}, \mathbb{R}^{d}\right), \quad t \in \mathbb{R}_{+}, \quad n \in \mathbb{N}$. Further, by (6.14), $\mathcal{X}=\Psi(\mathcal{M})$, where the mapping $\Psi: \mathbb{D}\left(\mathbb{R}_{+}, \mathbb{R}^{d}\right) \rightarrow \mathbb{D}\left(\mathbb{R}_{+}, \mathbb{R}^{d}\right)$ is given by

$$
\Psi(f)(t):=\Pi\left(f(t)+\stackrel{\widetilde{\widetilde{\beta}}}{)}, \quad f \in \mathbb{D}\left(\mathbb{R}_{+}, \mathbb{R}^{d}\right), \quad t \in \mathbb{R}_{+} .\right.
$$

Measurability of the mappings $\Psi_{n}, n \in \mathbb{N}$, and $\Psi$ can be checked similarly as in Barczy et al. [3, page 603]. We only note that, with the notations of Barczy et al. [3], for all $n, N \in \mathbb{N}$, the mappings $\psi_{n}^{N, 1}: \mathbb{D}\left(\mathbb{R}_{+}, \mathbb{R}^{d}\right) \rightarrow \mathbb{R}^{(n N+1) d}$ and $\psi_{n}^{N, 2}: \mathbb{R}^{(n N+1) d} \rightarrow \mathbb{D}\left(\mathbb{R}_{+}, \mathbb{R}^{d}\right)$ should be defined by

$$
\begin{aligned}
& \psi_{n}^{N, 1}(f):=\left(f(0), f\left(\frac{1}{n}\right), f\left(\frac{2}{n}\right), \ldots, f(N)\right), \\
& \psi_{n}^{N, 2}\left(\boldsymbol{x}_{0}, \boldsymbol{x}_{1}, \ldots, \boldsymbol{x}_{n N}\right)(t):=\mathrm{e}^{\lfloor n t] \widetilde{\boldsymbol{B}}} \boldsymbol{x}_{0}+\sum_{j=1}^{\lfloor n t\rfloor} \mathrm{e}^{(\lfloor n t\rfloor-j) \widetilde{\boldsymbol{B}}}\left(\boldsymbol{x}_{j}-\boldsymbol{x}_{j-1}+n^{-1} \widetilde{\widetilde{\beta}}\right)
\end{aligned}
$$

for $f \in \mathbb{D}\left(\mathbb{R}_{+}, \mathbb{R}^{d}\right), t \in \mathbb{R}_{+}$and $\left(\boldsymbol{x}_{0}^{\top}, \boldsymbol{x}_{1}^{\top}, \ldots, \boldsymbol{x}_{n N}^{\top}\right)^{\top} \in \mathbb{R}^{(n N+1) d}$.

The aim of the following discussion is to show that the set $C:=\left\{f \in \mathbb{C}\left(\mathbb{R}_{+}, \mathbb{R}^{d}\right): \Pi f(0)=\right.$ $f(0)\}$ satisfies $C \in \mathcal{D}_{\infty}\left(\mathbb{R}_{+}, \mathbb{R}^{d}\right), C \subset C_{\Psi,\left(\Psi_{n}\right)_{n \in \mathbb{N}}}$ and $\mathbb{P}(\mathcal{M} \in C)=1$.

First note that $C=\mathbb{C}\left(\mathbb{R}_{+}, \mathbb{R}^{d}\right) \cap \pi_{0}^{-1}\left(\left\{\boldsymbol{x} \in \mathbb{R}^{d}:\left(\boldsymbol{I}_{d}-\boldsymbol{\Pi}\right) \boldsymbol{x}=\mathbf{0}\right\}\right)$, where $\pi_{0}: \mathbb{D}\left(\mathbb{R}_{+}, \mathbb{R}^{d}\right) \rightarrow$ $\mathbb{R}^{d}$ denotes the projection defined by $\pi_{0}(f):=f(0)$ for $f \in \mathbb{D}\left(\mathbb{R}_{+}, \mathbb{R}^{d}\right)$. Using that $\mathbb{C}\left(\mathbb{R}_{+}, \mathbb{R}^{d}\right) \in \mathcal{D}_{\infty}\left(\mathbb{R}_{+}, \mathbb{R}^{d}\right)$ (see, e.g., Ethier and Kurtz [11, Problem 3.11.25]), the mapping $\mathbb{R}^{d} \ni \boldsymbol{x} \mapsto\left(\boldsymbol{I}_{d}-\boldsymbol{\Pi}\right) \boldsymbol{x} \in \mathbb{R}^{d}$ is measurable and that $\pi_{0}$ is measurable (see, e.g., Ethier and Kurtz [11, Proposition 3.7.1]), we obtain $C \in \mathcal{D}_{\infty}\left(\mathbb{R}_{+}, \mathbb{R}^{d}\right)$.

Fix a function $f \in C$ and a sequence $\left(f_{n}\right)_{n \in \mathbb{N}}$ in $\mathbb{D}\left(\mathbb{R}_{+}, \mathbb{R}^{d}\right)$ with $f_{n} \stackrel{\text { lu }}{\longrightarrow} f$ as $n \rightarrow \infty$. By the definition of $\Psi$, we have $\Psi(f) \in \mathbb{C}\left(\mathbb{R}_{+}, \mathbb{R}^{d}\right)$. Furthermore,

$$
\begin{aligned}
\Psi_{n}\left(f_{n}\right)(t)= & \Pi\left(f_{n}\left(\frac{\lfloor n t\rfloor}{n}\right)+\frac{\lfloor n t\rfloor}{n} \widetilde{\boldsymbol{\beta}}\right)+\left(\mathrm{e}^{\lfloor n t\rfloor \widetilde{\boldsymbol{B}}}-\boldsymbol{\Pi}\right) f_{n}(0) \\
& +\sum_{j=1}^{\lfloor n t\rfloor}\left(\mathrm{e}^{(\lfloor n t\rfloor-j) \widetilde{\boldsymbol{B}}}-\boldsymbol{\Pi}\right)\left(f_{n}\left(\frac{j}{n}\right)-f_{n}\left(\frac{j-1}{n}\right)+\frac{1}{n} \widetilde{\boldsymbol{\beta}}\right),
\end{aligned}
$$


hence we have for all $t \in \mathbb{R}_{+}$,

$$
\begin{aligned}
\left\|\Psi_{n}\left(f_{n}\right)(t)-\Psi(f)(t)\right\| \leqslant & \|\boldsymbol{\Pi}\|\left(\left\|f_{n}\left(\frac{\lfloor n t\rfloor}{n}\right)-f(t)\right\|+\frac{1}{n}\|\widetilde{\widetilde{\boldsymbol{\beta}}}\|\right)+\left\|\left(\mathrm{e}^{\lfloor n t\rfloor \widetilde{\boldsymbol{B}}}-\boldsymbol{\Pi}\right) f_{n}(0)\right\| \\
& +\sum_{j=1}^{\lfloor n t\rfloor}\left\|\mathrm{e}^{(\lfloor n t\rfloor-j) \widetilde{\boldsymbol{B}}}-\boldsymbol{\Pi}\right\|\left(\left\|f_{n}\left(\frac{j}{n}\right)-f_{n}\left(\frac{j-1}{n}\right)\right\|+\frac{1}{n}\|\widetilde{\boldsymbol{\beta}}\|\right) .
\end{aligned}
$$

Here for all $T>0$ and $t \in[0, T]$,

$$
\begin{aligned}
\left\|f_{n}\left(\frac{\lfloor n t\rfloor}{n}\right)-f(t)\right\| & \leqslant\left\|f_{n}\left(\frac{\lfloor n t\rfloor}{n}\right)-f\left(\frac{\lfloor n t\rfloor}{n}\right)\right\|+\left\|f\left(\frac{\lfloor n t\rfloor}{n}\right)-f(t)\right\| \\
& \leqslant \sup _{t \in[0, T]}\left\|f_{n}(t)-f(t)\right\|+\omega_{T}\left(f, n^{-1}\right),
\end{aligned}
$$

where $\omega_{T}(f, \cdot)$ is the modulus of continuity of $f$ on $[0, T]$, and we have $\omega_{T}\left(f, n^{-1}\right) \rightarrow 0$ since $f$ is continuous (see, e.g., Jacod and Shiryaev [19, VI.1.6]). In a similar way, for all $T>0$,

$$
\left\|f_{n}\left(\frac{j}{n}\right)-f_{n}\left(\frac{j-1}{n}\right)\right\| \leqslant \omega_{T}\left(f, n^{-1}\right)+2 \sup _{t \in[0, T]}\left\|f_{n}(t)-f(t)\right\|, \quad j \in\{1, \ldots,\lfloor n T\rfloor\} .
$$

By (iv) of Lemma A.3, for all $T>0$ and $t \in[0, T]$,

$$
\sum_{j=1}^{\lfloor n t\rfloor}\left\|\mathrm{e}^{(\lfloor n t\rfloor-j) \widetilde{B}}-\Pi\right\| \leqslant \sum_{j=1}^{\lfloor n t\rfloor} c \mathrm{e}^{-(\lfloor n t\rfloor-j) \kappa} \leqslant \frac{c}{1-\mathrm{e}^{-\kappa}} .
$$

Further, for all $T>0$ and $t \in[0, T]$, by (iv) of Lemma A.3,

$$
\begin{aligned}
\left\|\left(\mathrm{e}^{\lfloor n t] \widetilde{\boldsymbol{B}}}-\Pi\right) f_{n}(0)\right\| & \leqslant\left\|\left(\mathrm{e}^{\lfloor n t] \widetilde{\boldsymbol{B}}}-\Pi\right)\left(f_{n}(0)-f(0)\right)\right\|+\left\|\left(\mathrm{e}^{\lfloor n t] \widetilde{\boldsymbol{B}}}-\Pi\right) f(0)\right\| \\
& \leqslant c \sup _{t \in[0, T]}\left\|f_{n}(t)-f(t)\right\|,
\end{aligned}
$$

since $f \in C$ implies $\left(\mathrm{e}^{\lfloor n t] \widetilde{B}}-\boldsymbol{\Pi}\right) f(0)=\mathbf{0}$. Indeed, by part (iii) of Lemma A.3. $\mathrm{e}^{\lfloor n t] \widetilde{B}} \boldsymbol{\Pi}=$ $\mathrm{e}^{\lfloor n t\rfloor \widetilde{B}} \lim _{s \rightarrow \infty} \mathrm{e}^{\mathrm{s}^{\widetilde{B}}}=\lim _{s \rightarrow \infty} \mathrm{e}^{(\lfloor n t\rfloor+s) \widetilde{B}}=\Pi$, hence $\mathrm{e}^{\lfloor n t\rfloor \widetilde{B}} f(0)=\mathrm{e}^{\lfloor n t\rfloor \widetilde{B}} \Pi f(0)=\Pi f(0)$. Using that $f_{n} \stackrel{\mathrm{lu}}{\longrightarrow} f$ as $n \rightarrow \infty$, we have $\Psi_{n}\left(f_{n}\right) \stackrel{\mathrm{lu}}{\longrightarrow} \Psi(f)$ as $n \rightarrow \infty$. Thus we conclude $C \subset C_{\Psi,\left(\Psi_{n}\right)_{n \in \mathbb{N}}}$.

By the definition of a weak solution (see, e.g., Karatzas and Shreve [22, Definition 3.1, Section 5.3]), $\mathcal{M}$ has continuous sample paths almost surely, hence, since $\boldsymbol{\mathcal { M }}_{0}=\mathbf{0}$, we have $\mathbb{P}(\mathcal{M} \in C)=1$. Consequently, by Lemma D.1, we obtain $\mathcal{X}^{(n)}=\Psi_{n}\left(\mathcal{M}^{(n)}\right) \stackrel{\mathcal{D}}{\longrightarrow} \Psi(\mathcal{M})=\mathcal{X}$ as $n \rightarrow \infty$.

It remains to show that the limit process given by (6.14) coincides in law with the corresponding one in (4.2). Using $\Pi=\boldsymbol{u v}^{\top}$ and $\boldsymbol{v}^{\top} \boldsymbol{u}=1$, we get that the process $\mathcal{X}_{t}:=\boldsymbol{v}^{\top} \mathcal{X}_{t}$, $t \in \mathbb{R}_{+}$(where $\left(\mathcal{X}_{t}\right)_{t \in \mathbb{R}_{+}}$is given by $(6.14)$ ) satisfies

$$
\mathcal{X}_{t}=\boldsymbol{v}^{\top} \boldsymbol{\Pi}\left(\mathcal{M}_{t}+t \widetilde{\widetilde{\beta}}\right)=\boldsymbol{v}^{\top}\left(\mathcal{M}_{t}+t \widetilde{\widetilde{\beta}}\right), \quad t \in \mathbb{R}_{+},
$$


hence $\mathcal{X}_{t} \boldsymbol{u}=\boldsymbol{u} \boldsymbol{v}^{\top}\left(\mathcal{M}_{t}+t \widetilde{\widetilde{\boldsymbol{\beta}}}\right)=\Pi\left(\mathcal{M}_{t}+t \widetilde{\widetilde{\boldsymbol{\beta}}}\right)=\mathcal{X}_{t}, \quad t \in \mathbb{R}_{+}$. By (6.4) and Itô's formula we obtain that $\left(\mathcal{X}_{t}\right)_{t \in \mathbb{R}_{+}}$is a strong solution of the SDE

$$
\mathrm{d} \mathcal{X}_{t}=\boldsymbol{v}^{\top} \widetilde{\widetilde{\beta}} \mathrm{d} t+\sqrt{\mathcal{X}_{t}^{+}} \boldsymbol{v}^{\top} \sqrt{\widetilde{\boldsymbol{C}}} \mathrm{d} \mathcal{W}_{t}, \quad t \in \mathbb{R}_{+}, \quad \mathcal{X}_{0}=0
$$

where $\left(\mathcal{W}_{t}\right)_{t \in \mathbb{R}_{+}}$is a $d$-dimensional standard Brownian motion. This equation can be written in the form

$$
\mathrm{d} \mathcal{X}_{t}=\boldsymbol{v}^{\top} \widetilde{\widetilde{\beta}} \mathrm{d} t+\sqrt{\boldsymbol{v}^{\top} \widetilde{\boldsymbol{C}} \boldsymbol{v} \mathcal{X}_{t}^{+}} \mathrm{d} \mathcal{W}_{t}, \quad t \in \mathbb{R}_{+}, \quad \mathcal{X}_{0}=0,
$$

with some 1-dimensional standard Brownian motion $\left(\mathcal{W}_{t}\right)_{t \in \mathbb{R}_{+}}$. Indeed, $\boldsymbol{v}^{\top} \widetilde{\boldsymbol{C}} \boldsymbol{v}=$ $\left(\boldsymbol{v}^{\top} \sqrt{\widetilde{\boldsymbol{C}}}\right)\left(\boldsymbol{v}^{\top} \sqrt{\widetilde{\boldsymbol{C}}}\right)^{\top}=\left\|\boldsymbol{v}^{\top} \sqrt{\widetilde{\boldsymbol{C}}}\right\|^{2}$, and hence if $\boldsymbol{v}^{\top} \sqrt{\widetilde{\boldsymbol{C}}}=\mathbf{0} \in \mathbb{R}^{1 \times d}$, then the above mentioned rewriting of the SDE in question is trivial, and if $\boldsymbol{v}^{\top} \sqrt{\widetilde{\boldsymbol{C}}} \neq \mathbf{0} \in \mathbb{R}^{1 \times d}$, then $\mathcal{W}_{t}:=\left(\boldsymbol{v}^{\top} \widetilde{\boldsymbol{C}} \boldsymbol{v}\right)^{-1 / 2} \boldsymbol{v}^{\top} \sqrt{\widetilde{\boldsymbol{C}}} \mathcal{W}_{t}, \quad t \in \mathbb{R}_{+}$, is a 1-dimensional standard Brownian motion. Finally, the SDE (6.15) can be written in the form (4.3), since

$$
\boldsymbol{v}^{\top} \widetilde{\widetilde{\beta}}=\int_{0}^{1} \boldsymbol{v}^{\top} \mathrm{e}^{s \widetilde{\boldsymbol{B}}} \widetilde{\boldsymbol{\beta}} \mathrm{d} s=\int_{0}^{1} \boldsymbol{v}^{\top} \widetilde{\boldsymbol{\beta}} \mathrm{d} s=\boldsymbol{v}^{\top} \widetilde{\boldsymbol{\beta}},
$$

and

$$
\begin{aligned}
\langle\widetilde{\boldsymbol{C}} \boldsymbol{v}, \boldsymbol{v}\rangle=\boldsymbol{v}^{\top} \widetilde{\boldsymbol{C}} \boldsymbol{v} & =\sum_{k=1}^{d}\left(\boldsymbol{e}_{k}^{\top} \boldsymbol{u}\right) \int_{0}^{1} \boldsymbol{v}^{\top} \mathrm{e}^{s \widetilde{\boldsymbol{B}}} \boldsymbol{C}_{k} \mathrm{e}^{s \widetilde{\boldsymbol{B}}^{\top}} \boldsymbol{v} \mathrm{d} s=\sum_{k=1}^{d}\left(\boldsymbol{e}_{k}^{\top} \boldsymbol{u}\right) \int_{0}^{1} \boldsymbol{v}^{\top} \boldsymbol{C}_{k} \boldsymbol{v} \mathrm{d} s \\
& =\sum_{k=1}^{d}\left(\boldsymbol{e}_{k}^{\top} \boldsymbol{u}\right) \boldsymbol{v}^{\top} \boldsymbol{C}_{k} \boldsymbol{v}=\boldsymbol{v}^{\top}\left(\sum_{k=1}^{d}\left(\boldsymbol{e}_{k}^{\top} \boldsymbol{u}\right) \boldsymbol{C}_{k}\right) \boldsymbol{v}=\boldsymbol{v}^{\top} \overline{\boldsymbol{C}} \boldsymbol{v}=\langle\overline{\boldsymbol{C}} \boldsymbol{v}, \boldsymbol{v}\rangle
\end{aligned}
$$

Hence $\left(\mathcal{X}_{t}\right)_{t \in \mathbb{R}_{+}}$is a strong solution of the SDE (4.3) and consequently, we conclude (4.2).

\section{Appendices}

\section{A Frobenius-Perron type results}

For the classification of CBI processes and for the estimation of the moments of a CBI process $\left(\boldsymbol{X}_{t}\right)_{t \in \mathbb{R}_{+}}$, we need some Frobenius-Perron type statements about the asymptotic behaviour of $\mathrm{e}^{t \boldsymbol{A}}$ as $t \rightarrow \infty$, where $\boldsymbol{A}=\left(a_{i, j}\right)_{i, j \in\{1, \ldots, d\}} \in \mathbb{R}_{(+)}^{d \times d}$, i.e., $\boldsymbol{A}$ is essentially non-negative. Note that then $\mathrm{e}^{\boldsymbol{A}} \in \mathbb{R}_{+}^{d \times d}$. Indeed, we have $\boldsymbol{A}-a_{\boldsymbol{A}} \boldsymbol{I}_{d} \in \mathbb{R}_{+}^{d \times d}$ with $a_{\boldsymbol{A}}:=\min _{i \in\{1, \ldots, d\}} a_{i, i}$, thus $\mathrm{e}^{\boldsymbol{A}}=\mathrm{e}^{a_{\boldsymbol{A}}} \mathrm{e}^{\boldsymbol{A}-a_{\boldsymbol{A}} \boldsymbol{I}_{d}} \in \mathbb{R}_{+}^{d \times d}$. Recall that $s(\boldsymbol{A})=\max _{\lambda \in \sigma(\boldsymbol{A})} \operatorname{Re}(\lambda)$, where $\sigma(\boldsymbol{A})$ denotes the spectrum of $\boldsymbol{A}$, i.e., the set of the eigenvalues of $\boldsymbol{A}$.

A.1 Lemma. Suppose that $\boldsymbol{A} \in \mathbb{R}_{(+)}^{d \times d}$. Then the following statements are equivalent: 
(i) there exists $t_{0} \in \mathbb{R}_{++}$such that $\mathrm{e}^{t_{0} A} \in \mathbb{R}_{++}^{d \times d}$;

(ii) for all $t \in \mathbb{R}_{++}$, we have $\mathrm{e}^{t \boldsymbol{A}} \in \mathbb{R}_{++}^{d \times d}$;

(iii) $\boldsymbol{A}$ is irreducible.

Proof. If $d=1$, then the statement is trivial. If $d \geqslant 2$, then the statement follows by Berman and Plemmons [7, Chapter 6, Theorem 3.12]. However, for the claim (i) $\Longrightarrow$ (ii) we give an independent proof, which may be interesting on its own. Let $\widetilde{\boldsymbol{A}}=\left(\widetilde{a}_{i, j}\right)_{i, j \in\{1, \ldots, d\}}:=$ $\boldsymbol{A}-a_{\boldsymbol{A}} \boldsymbol{I}_{d} \in \mathbb{R}_{+}^{d \times d}$ and $K:=1+\max _{i \in\{1, \ldots, d\}} \boldsymbol{e}_{i}^{\top} \widetilde{\boldsymbol{A}} \mathbf{1} \in \mathbb{R}_{++}$, where $\mathbf{1}:=(1, \ldots, 1)^{\top} \in \mathbb{R}^{d}$. Then the matrix $\boldsymbol{Q}=\left(q_{i, j}\right)_{i, j \in\{1, \ldots, d+1\}} \in \mathbb{R}_{+}^{(d+1) \times(d+1)}$, given by

$$
q_{i, j}:= \begin{cases}K^{-1} \widetilde{a}_{i, j}, & \text { if } i, j \in\{1, \ldots, d\}, \\ 1-K^{-1} \boldsymbol{e}_{i}^{\top} \widetilde{\boldsymbol{A}} 1, & \text { if } i \in\{1, \ldots, d\} \text { and } j=d+1, \\ 0, & \text { if } i=d+1 \text { and } j \in\{1, \ldots, d\}, \\ 1, & \text { if } i=d+1 \text { and } j=d+1,\end{cases}
$$

is a stochastic matrix, since the entries are non-negative and $\widetilde{\boldsymbol{e}}_{i}^{\top} \boldsymbol{Q} \widetilde{\mathbf{1}}=1$ for all $i \in\{1, \ldots, d+$ $1\}$, where $\widetilde{\boldsymbol{e}}_{1}, \ldots, \widetilde{\boldsymbol{e}}_{d+1}$ denotes the natural basis in $\mathbb{R}^{d+1}$, and $\widetilde{\mathbf{1}}:=(1, \ldots, 1)^{\top} \in \mathbb{R}^{d+1}$. Indeed, for each $i \in\{1, \ldots, d\}$, we have $\widetilde{\boldsymbol{e}}_{i}^{\top} \boldsymbol{Q} \widetilde{\mathbf{1}}=\boldsymbol{e}_{i}^{\top} K^{-1} \widetilde{\boldsymbol{A}} \mathbf{1}+\left(1-K^{-1} \boldsymbol{e}_{i}^{\top} \widetilde{\boldsymbol{A}} \mathbf{1}\right)=1$, and $\widetilde{\boldsymbol{e}}_{d+1}^{\top} \boldsymbol{Q} \widetilde{\mathbf{1}}=q_{d+1, d+1}=1$. By Chung [8, Theorem II.1.5], for all $i, j \in\{1, \ldots, d+1\}$, there are two possibilities, namely, either $\widetilde{\boldsymbol{e}}_{i}^{\top} \mathrm{e}^{t \boldsymbol{Q}} \widetilde{\boldsymbol{e}}_{j}>0$ for all $t \in \mathbb{R}_{++}$, or $\widetilde{\boldsymbol{e}}_{i}^{\top} \mathrm{e}^{t \boldsymbol{Q}} \widetilde{\boldsymbol{e}}_{j}=0$ for all $t \in \mathbb{R}_{++}$. Clearly, $\widetilde{a}_{i, j}=K q_{i, j}, i, j \in\{1, \ldots, d\}$, and $\widetilde{\boldsymbol{e}}_{d+1}^{\top} \boldsymbol{Q} \widetilde{\boldsymbol{e}}_{j}=0, j \in\{1, \ldots, d\}$, imply $\boldsymbol{e}_{i}^{\top} \widetilde{\boldsymbol{A}}^{n} \boldsymbol{e}_{j}=K^{n} \widetilde{\boldsymbol{e}}_{i}^{\top} \boldsymbol{Q}^{n} \widetilde{\boldsymbol{e}}_{j}$ for all $i, j \in\{1, \ldots, d\}$ and $n \in \mathbb{N}$. Indeed, this obviously holds for $n=1$, and, by induction,

$$
\begin{aligned}
\boldsymbol{e}_{i}^{\top} \widetilde{\boldsymbol{A}}^{n+1} \boldsymbol{e}_{j} & =\sum_{k=1}^{d}\left(\boldsymbol{e}_{i}^{\top} \widetilde{\boldsymbol{A}}^{n} \boldsymbol{e}_{k}\right)\left(\boldsymbol{\boldsymbol { e } _ { k } ^ { \top }} \widetilde{\boldsymbol{A}} \boldsymbol{e}_{j}\right)=K^{n+1} \sum_{k=1}^{d}\left(\widetilde{\boldsymbol{e}}_{i}^{\top} \boldsymbol{Q}^{n} \widetilde{\boldsymbol{e}}_{k}\right)\left(\widetilde{\boldsymbol{e}}_{k}^{\top} \boldsymbol{Q} \widetilde{\boldsymbol{e}}_{j}\right) \\
& =K^{n+1} \sum_{k=1}^{d+1}\left(\widetilde{\boldsymbol{e}}_{i}^{\top} \boldsymbol{Q}^{n} \widetilde{\boldsymbol{e}}_{k}\right)\left(\widetilde{\boldsymbol{e}}_{k}^{\top} \boldsymbol{Q} \widetilde{\boldsymbol{e}}_{j}\right)=K^{n+1} \widetilde{\boldsymbol{e}}_{i}^{\top} \boldsymbol{Q}^{n} \widetilde{\boldsymbol{e}}_{j}
\end{aligned}
$$

Consequently,

$$
\boldsymbol{e}_{i}^{\top} \mathrm{e}^{t \widetilde{\boldsymbol{A}}} \boldsymbol{e}_{j}=\boldsymbol{e}_{i}^{\top} \sum_{n=0}^{\infty} \frac{t^{n} \widetilde{\boldsymbol{A}}^{n}}{n !} \boldsymbol{e}_{j}=\widetilde{\boldsymbol{e}}_{i}^{\top} \sum_{n=0}^{\infty} \frac{K^{n} t^{n} \boldsymbol{Q}^{n}}{n !} \widetilde{\boldsymbol{e}}_{j}=\widetilde{\boldsymbol{e}}_{i}^{\top} \mathrm{e}^{K t \boldsymbol{Q}} \widetilde{\boldsymbol{e}}_{j}
$$

for all $i, j \in\{1, \ldots, d\}$. Now the assumption $\mathrm{e}^{t_{0} \boldsymbol{A}} \in \mathbb{R}_{++}^{d \times d}$ implies $\widetilde{\boldsymbol{e}}_{i}^{\top} \mathrm{e}^{K t_{0} \boldsymbol{Q}} \widetilde{\boldsymbol{e}}_{j}=\boldsymbol{e}_{i}^{\top} \mathrm{e}^{t_{0}} \widetilde{\boldsymbol{A}}_{\boldsymbol{e}} \boldsymbol{e}_{j}=$ $\mathrm{e}^{-t_{0} a_{\boldsymbol{A}}} \boldsymbol{e}_{i}^{\top} \mathrm{e}^{t_{0} \boldsymbol{A}} \boldsymbol{e}_{j}>0$ for all $i, j \in\{1, \ldots, d\}$, hence, by Chung [8, Theorem II.1.5], $\boldsymbol{e}_{i}^{\top} \mathrm{e}^{t \widetilde{\boldsymbol{A}}} \boldsymbol{e}_{j}=$ $\widetilde{\boldsymbol{e}}_{i}^{\top} \mathrm{e}^{K t \boldsymbol{Q}} \widetilde{\boldsymbol{e}}_{j}>0$ for all $i, j \in\{1, \ldots, d\}$ and $t \in \mathbb{R}_{++}$, and we conclude (ii).

A.2 Remark. Exercise 7.7.4 of the Internet Seminar [1] claims that the above statements are equivalent to irreducibility of the matrix $\mathrm{e}^{t \boldsymbol{A}}$ for some or for any $t \in \mathbb{R}_{++}$. Thus a multi-type CBI process is irreducible if and only if $\mathrm{e}^{\widetilde{B}}$ is irreducible. 
A.3 Lemma. Suppose that $\boldsymbol{A} \in \mathbb{R}_{(+)}^{d \times d}$ is irreducible.

(i) Then $s(\boldsymbol{A})$ is an eigenvalue of $\boldsymbol{A}$, the algebraic and geometric multiplicities of $s(\boldsymbol{A})$ equal 1, and the real parts of the other eigenvalues of $\boldsymbol{A}$ are less than $s(\boldsymbol{A})$.

(ii) Corresponding to the eigenvalue $s(\boldsymbol{A})$ there exists a unique (right) eigenvector $\boldsymbol{u}_{\boldsymbol{A}} \in \mathbb{R}_{++}^{d}$ of $\boldsymbol{A}$ such that the sum of its coordinates is 1. The vector $\boldsymbol{u}_{\boldsymbol{A}} \in \mathbb{R}_{++}^{d}$ is the unique (right) eigenvector of $\mathrm{e}^{\boldsymbol{A}}$ (called the right Perron vector of $\mathrm{e}^{\boldsymbol{A}}$ ) corresponding to the eigenvalue $r\left(\mathrm{e}^{\boldsymbol{A}}\right)=\mathrm{e}^{s(\boldsymbol{A})}$ of $\mathrm{e}^{\boldsymbol{A}}$ such that the sum of its coordinates is 1.

(iii) There exists a unique left eigenvector $\boldsymbol{v}_{\boldsymbol{A}} \in \mathbb{R}_{++}^{d}$ of $\boldsymbol{A}$ corresponding to the eigenvalue $s(\boldsymbol{A})$ with $\boldsymbol{u}_{\boldsymbol{A}}^{\top} \boldsymbol{v}_{\boldsymbol{A}}=1$, and

$$
\mathrm{e}^{-s(\boldsymbol{A}) t} \mathrm{e}^{t \boldsymbol{A}} \rightarrow \boldsymbol{\Pi}_{\boldsymbol{A}}:=\boldsymbol{u}_{\boldsymbol{A}} \boldsymbol{v}_{\boldsymbol{A}}^{\top} \in \mathbb{R}_{++}^{d \times d} \quad \text { as } t \rightarrow \infty .
$$

The vector $\boldsymbol{v}_{\boldsymbol{A}} \in \mathbb{R}_{++}^{d}$ is the unique (left) eigenvector of $\mathrm{e}^{\boldsymbol{A}}$ (called the left Perron vector of $\mathrm{e}^{\boldsymbol{A}}$ ) corresponding to the eigenvalue $r\left(\mathrm{e}^{\boldsymbol{A}}\right)=\mathrm{e}^{s(\boldsymbol{A})}$ of $\mathrm{e}^{\boldsymbol{A}}$ such that $\boldsymbol{u}_{\boldsymbol{A}}^{\top} \boldsymbol{v}_{\boldsymbol{A}}=1$.

(iv) There exist $c_{\boldsymbol{A}}, \kappa_{\boldsymbol{A}} \in \mathbb{R}_{++}$such that

$$
\left\|\mathrm{e}^{-s(\boldsymbol{A}) t} \mathrm{e}^{t \boldsymbol{A}}-\boldsymbol{\Pi}_{\boldsymbol{A}}\right\| \leqslant c_{\boldsymbol{A}} \mathrm{e}^{-\kappa_{\boldsymbol{A}} t} \quad \text { for all } t \in \mathbb{R}_{+} .
$$

Consequently, $\left\|\mathrm{e}^{t \boldsymbol{A}}\right\| \leqslant\left(c_{\boldsymbol{A}}+\left\|\boldsymbol{\Pi}_{\boldsymbol{A}}\right\|\right) \mathrm{e}^{-s(\boldsymbol{A}) t}, \quad t \in \mathbb{R}_{+}$.

(v) Moreover,

$$
\frac{1}{t} \int_{0}^{t} \mathrm{e}^{-s(\boldsymbol{A}) u} \mathrm{e}^{u \boldsymbol{A}} \mathrm{d} u \rightarrow \boldsymbol{\Pi}_{\boldsymbol{A}} \quad \text { as } t \rightarrow \infty .
$$

Proof. The proof of (i) is based on the Frobenius-Perron theorem for $\mathrm{e}^{\boldsymbol{A}}$ (see, e.g., Horn and Johnson [12, Theorems 8.2.11 and 8.5.1]). Recall that $\sigma\left(\mathrm{e}^{\boldsymbol{A}}\right)=\mathrm{e}^{\sigma(\boldsymbol{A})}$ and $r\left(\mathrm{e}^{\boldsymbol{A}}\right)=\mathrm{e}^{s(\boldsymbol{A})}$, see (3.1). By Lemma A.1, $\mathrm{e}^{\boldsymbol{A}} \in \mathbb{R}_{++}^{d \times d}$, hence, by the Frobenius-Perron theorem, $r\left(\mathrm{e}^{\boldsymbol{A}}\right) \in \mathbb{R}_{++}$ is an eigenvalue of $\mathrm{e}^{\boldsymbol{A}}$, the algebraic and geometric multiplicities of $r\left(\mathrm{e}^{\boldsymbol{A}}\right)$ equal 1 , and the absolute values of the other eigenvalues of $\mathrm{e}^{\boldsymbol{A}}$ are less than $r\left(\mathrm{e}^{\boldsymbol{A}}\right)$. Then $s(\boldsymbol{A})=\log \left[r\left(\mathrm{e}^{\boldsymbol{A}}\right)\right]$ is an eigenvalue of $\boldsymbol{A}$, the algebraic and geometric multiplicities of $s(\boldsymbol{A})$ equal 1, and the real parts of the other eigenvalues of $\boldsymbol{A}$ are less than $s(\boldsymbol{A})$. Indeed, since $\sigma\left(\mathrm{e}^{\boldsymbol{A}}\right)=\mathrm{e}^{\sigma(\boldsymbol{A})}$, the algebraic multiplicity of $s(\boldsymbol{A})$ (as an eigenvalue of $\boldsymbol{A}$ ) coincides with the algebraic multiplicity of $r\left(\mathrm{e}^{\boldsymbol{A}}\right)$ (as an eigenvalue of $\mathrm{e}^{\boldsymbol{A}}$ ), yielding that the algebraic multiplicity of $s(\boldsymbol{A})$ is 1 ; and using that the geometric multiplicity of $s(\boldsymbol{A})$ is less than or equal to its algebraic multiplicity, we obtain that the geometric multiplicity of $s(\boldsymbol{A})$ equals 1 , too. Further, if $\lambda$ is an eigenvalue of $\boldsymbol{A}$ not equal to $s(\boldsymbol{A})$, then $\mathrm{e}^{\lambda}$ is an eigenvalue of $\mathrm{e}^{\boldsymbol{A}}$ and $r\left(\mathrm{e}^{\boldsymbol{A}}\right)>\left|\mathrm{e}^{\lambda}\right|=\mathrm{e}^{\operatorname{Re}(\lambda)}$ yields that $s(\boldsymbol{A})=\log \left(r\left(\mathrm{e}^{\boldsymbol{A}}\right)\right)>\operatorname{Re}(\lambda)$, as it was stated, hence the proof of (i) is complete.

The Frobenius-Perron theorem also implies (ii) and the unique existence of $\boldsymbol{v}_{\boldsymbol{A}}$ in (iii), and the convergence in (iii) along the sequence of the positive integers. The aim of the following 
discussion is to show that the convergence in (iii) holds also along the positive real numbers. By Dunford and Schwartz [10, Theorem VII.1.8],

$$
\mathrm{e}^{t \boldsymbol{A}}=\sum_{\lambda \in \sigma(\boldsymbol{A})} \sum_{i=0}^{\nu(\lambda)-1} \frac{\left(\boldsymbol{A}-\lambda \boldsymbol{I}_{d}\right)^{i}}{i !} t^{i} \mathrm{e}^{\lambda t} E_{\boldsymbol{A}}(\lambda), \quad t \in \mathbb{R}_{+},
$$

where $\nu(\lambda)$ denotes the index of $\lambda$ given in Dunford and Schwartz [10, Definition VII.1.2], and the projections $E_{\boldsymbol{A}}(\lambda), \lambda \in \sigma(\boldsymbol{A})$, are defined by $E_{\boldsymbol{A}}(\lambda):=e_{\boldsymbol{A}, \lambda}(\boldsymbol{A})$, where $e_{\boldsymbol{A}, \lambda}: \mathbb{C} \rightarrow \mathbb{C}$ is an analytic function in some open set containing $\sigma(\boldsymbol{A})$ such that $e_{\boldsymbol{A}, \lambda}(\mu)=1$ if $\mu$ is in some neighborhood of $\lambda$ and $e_{\boldsymbol{A}, \lambda}(\mu)=0$ if $\mu$ is in some neighborhood of any point of $\sigma(\boldsymbol{A}) \backslash\{\lambda\}$, see the definition of the function $e_{\boldsymbol{A}, \lambda}$ in Dunford and Schwartz [10, before Theorem VII.1.6]. Here $e_{\boldsymbol{A}, \lambda}(\boldsymbol{A})=P_{\boldsymbol{A}, \lambda}(\boldsymbol{A})$, where $P_{\boldsymbol{A}, \lambda}: \mathbb{C} \rightarrow \mathbb{C}$ is a polynomial with complex coefficients such that $P_{\boldsymbol{A}, \lambda}^{(m)}(\mu)=e_{\boldsymbol{A}, \lambda}^{(m)}(\mu)$ for all $\mu \in \sigma(\boldsymbol{A})$ and for all $m \in\{0,1, \ldots, \nu(\mu)-1\}$, see the definition of the matrix $e_{\boldsymbol{A}, \lambda}(\boldsymbol{A})$ in Dunford and Schwartz [10, before Theorem VII.1.5]. Consequently,

$$
\mathrm{e}^{t \boldsymbol{A}}=\sum_{\lambda \in \sigma(\boldsymbol{A})} \sum_{i=0}^{\nu(\lambda)-1} \frac{\left(\boldsymbol{A}-\lambda \boldsymbol{I}_{d}\right)^{i}}{i !} t^{i} \mathrm{e}^{\lambda t} P_{\boldsymbol{A}, \lambda}(\boldsymbol{A}), \quad t \in \mathbb{R}_{+}
$$

where $P_{\boldsymbol{A}, \lambda}$ is a polynomial with complex coefficients such that $P_{\boldsymbol{A}, \lambda}(\lambda)=1, \quad P_{\boldsymbol{A}, \lambda}^{(m)}(\lambda)=0$ for all $m \in\{1, \ldots, \nu(\lambda)-1\}$, and $P_{\boldsymbol{A}, \lambda}^{(m)}(\widetilde{\lambda})=0$ for all $\tilde{\lambda} \in \sigma(\boldsymbol{A})$ with $\tilde{\lambda} \neq \lambda$ and for all $m \in\{0,1, \ldots, \nu(\widetilde{\lambda})-1\}$.

Note that the index and the algebraic multiplicity of an eigenvalue $\lambda \in \sigma(\boldsymbol{A})$ coincide. Indeed, the index $\nu(\lambda)$ is the smallest non-negative integer $\nu \in \mathbb{Z}_{+}$such that $\mathcal{R}_{\lambda}^{(\nu+1)}=\mathcal{R}_{\lambda}^{(\nu)}$, where

$$
\mathcal{R}_{\lambda}^{(\nu)}:=\left\{\boldsymbol{x} \in \mathbb{C}^{d}:\left(\boldsymbol{A}-\lambda \boldsymbol{I}_{d}\right)^{\nu} \boldsymbol{x}=\mathbf{0}\right\} .
$$

By a change of basis of $\mathbb{R}^{d}$, we have $\boldsymbol{A}=\boldsymbol{S} \boldsymbol{J} \boldsymbol{S}^{-1}$ with some invertible matrix $\boldsymbol{S} \in \mathbb{C}^{d \times d}$, where $\boldsymbol{J}$ denotes the Jordan normal form of $\boldsymbol{A}$ consisting of Jordan blocks $\boldsymbol{J}_{\mu} \in \mathbb{C}^{m(\mu) \times m(\mu)}$, $\mu \in \sigma(\boldsymbol{A})$, where $m(\mu)$ is the algebraic multiplicity of $\mu$. One can easily verify that $\mathcal{R}_{\lambda}^{(\nu)}=\boldsymbol{S} \widetilde{\mathcal{R}}_{\lambda}^{(\nu)}$ for all $\nu \in \mathbb{Z}_{+}$, where

$$
\widetilde{\mathcal{R}}_{\lambda}^{(\nu)}:=\left\{\boldsymbol{x} \in \mathbb{C}^{d}:\left(\boldsymbol{J}-\lambda \boldsymbol{I}_{d}\right)^{\nu} \boldsymbol{x}=\mathbf{0}\right\} .
$$

For each $\mu \in \sigma(\boldsymbol{A})$ with $\mu \neq \lambda$, we have

$$
\left\{\boldsymbol{x} \in \mathbb{C}^{m(\mu)}:\left(\boldsymbol{J}_{\mu}-\lambda \boldsymbol{I}_{m(\mu)}\right)^{k} \boldsymbol{x}=\mathbf{0}\right\}=\{\mathbf{0}\}
$$

for all $k \in \mathbb{N}$, since $\left(\boldsymbol{J}_{\mu}-\lambda \boldsymbol{I}_{m(\mu)}\right)^{k}$ is invertible. Moreover, the dimension of the subspace

$$
\left\{\boldsymbol{x} \in \mathbb{C}^{m(\lambda)}:\left(\boldsymbol{J}_{\lambda}-\lambda \boldsymbol{I}_{m(\lambda)}\right)^{k} \boldsymbol{x}=\mathbf{0}\right\}
$$

equals $k$ for all $k \in\{1, \ldots, m(\lambda)\}$, hence $m(\lambda)$ is the smallest non-negative integer $\nu \in \mathbb{Z}_{+}$ such that $\widetilde{\mathcal{R}}_{\lambda}^{(\nu+1)}=\widetilde{\mathcal{R}}_{\lambda}^{(\nu)}$. Since $\mathcal{R}_{\lambda}^{(\nu)}=\boldsymbol{S} \widetilde{\mathcal{R}}_{\lambda}^{(\nu)}$ for all $\nu \in \mathbb{Z}_{+}$, we conclude $\nu(\lambda)=m(\lambda)$. 
By (i), the algebraic and geometric multiplicities of the eigenvalue $s(\boldsymbol{A})$ equal 1 , and hence we have $\nu(s(\boldsymbol{A}))=1$. Then $\mathrm{e}^{-s(\boldsymbol{A}) t} \mathrm{e}^{t \boldsymbol{A}} \rightarrow P_{\boldsymbol{A}, s(\boldsymbol{A})}(\boldsymbol{A})$ as $t \rightarrow \infty$, since, for each $\lambda \in \sigma(\boldsymbol{A})$ with $\lambda \neq s(\boldsymbol{A})$ we have $\operatorname{Re}(\lambda)<s(\boldsymbol{A})$, and then $\left|t^{i} \mathrm{e}^{-s(\boldsymbol{A}) t} \mathrm{e}^{\lambda t}\right|=t^{i} \mathrm{e}^{-(s(\boldsymbol{A})-\operatorname{Re}(\lambda)) t} \rightarrow 0$ as $t \rightarrow \infty$ for all $i \in\{0,1, \ldots, \nu(\lambda)-1\}$. Applying the Frobenius-Perron theorem for the matrix $\mathrm{e}^{\boldsymbol{A}}$, we obtain $\mathrm{e}^{-s(\boldsymbol{A}) n} \mathrm{e}^{n \boldsymbol{A}}=r\left(\mathrm{e}^{\boldsymbol{A}}\right)^{-n}\left(\mathrm{e}^{\boldsymbol{A}}\right)^{n} \rightarrow \boldsymbol{\Pi}_{\boldsymbol{A}}=\boldsymbol{u}_{\boldsymbol{A}} \boldsymbol{v}_{\boldsymbol{A}}^{\top}$ as $n \rightarrow \infty \quad$ (along the sequence of the positive integers), thus $P_{\boldsymbol{A}, s(\boldsymbol{A})}(\boldsymbol{A})=\boldsymbol{\Pi}_{\boldsymbol{A}}=\boldsymbol{u}_{\boldsymbol{A}} \boldsymbol{v}_{\boldsymbol{A}}^{\top}$, and we conclude (iii).

The statement (iv) is trivial for $d=1$. For $d \geqslant 2$, by formula (A.1), we obtain

$$
\left\|\mathrm{e}^{-s(\boldsymbol{A}) t} \mathrm{e}^{t \boldsymbol{A}}-\boldsymbol{\Pi}_{\boldsymbol{A}}\right\| \leqslant \sum_{\lambda \in \sigma(\boldsymbol{A}) \backslash\{s(\boldsymbol{A})\}} \sum_{i=0}^{\nu(\lambda)-1} \frac{\left\|\boldsymbol{A}-\lambda \boldsymbol{I}_{d}\right\|^{i}}{i !} t^{i}\left\|P_{\boldsymbol{A}, \lambda}(\boldsymbol{A})\right\| \mathrm{e}^{-(s(\boldsymbol{A})-\operatorname{Re}(\lambda)) t}
$$

for all $t \in \mathbb{R}_{+}$, hence we conclude (iv) with

$$
\begin{gathered}
\kappa_{\boldsymbol{A}}=\frac{1}{2}\left(s(\boldsymbol{A})-\max _{\lambda \in \sigma(\boldsymbol{A}) \backslash\{s(\boldsymbol{A})\}} \operatorname{Re}(\lambda)\right) \in \mathbb{R}_{++}, \\
c_{\boldsymbol{A}}=\sum_{\lambda \in \sigma(\boldsymbol{A}) \backslash\{s(\boldsymbol{A})\}} \sum_{i=0}^{\nu(\lambda)-1} \frac{\left\|\boldsymbol{A}-\lambda \boldsymbol{I}_{d}\right\|^{i}}{i !}\left\|P_{\boldsymbol{A}, \lambda}(\boldsymbol{A})\right\| \sup _{t \in \mathbb{R}_{+}}\left(t^{i} \mathrm{e}^{-\kappa_{\boldsymbol{A}} t}\right) \in \mathbb{R}_{++} .
\end{gathered}
$$

Indeed, $c_{\boldsymbol{A}}=0$ would lead us to a contradiction, since then, by $\sup _{t \in \mathbb{R}_{+}}\left(t^{i} \mathrm{e}^{-\kappa_{\boldsymbol{A}} t}\right)>0$, for all $\lambda \in \sigma(\boldsymbol{A}) \backslash\{s(\boldsymbol{A})\}$ and $i \in\{0, \ldots, \nu(\lambda)-1\}$, we get $\left\|\boldsymbol{A}-\lambda \boldsymbol{I}_{d}\right\|^{i}\left\|P_{\boldsymbol{A}, \lambda}(\boldsymbol{A})\right\|=0$, which implies $\left\|\mathrm{e}^{-s(\boldsymbol{A}) t} \mathrm{e}^{t \boldsymbol{A}}-\boldsymbol{\Pi}_{\boldsymbol{A}}\right\|=0$ for all $t \in \mathbb{R}_{+}$. With a special choice of $t=0$, this gives $\boldsymbol{\Pi}_{\boldsymbol{A}}=\boldsymbol{I}_{d}$, which is a contradiction, since $\boldsymbol{\Pi}_{\boldsymbol{A}} \in \mathbb{R}_{++}^{d \times d}$. Further,

$$
\left\|\mathrm{e}^{t \boldsymbol{A}}\right\|=\mathrm{e}^{s(\boldsymbol{A}) t}\left\|\mathrm{e}^{-s(\boldsymbol{A}) t} \mathrm{e}^{t \boldsymbol{A}}\right\| \leqslant \mathrm{e}^{s(\boldsymbol{A}) t}\left(\left\|\mathrm{e}^{-s(\boldsymbol{A}) t} \mathrm{e}^{t \boldsymbol{A}}-\boldsymbol{\Pi}_{\boldsymbol{A}}\right\|+\left\|\boldsymbol{\Pi}_{\boldsymbol{A}}\right\|\right) \leqslant\left(c_{\boldsymbol{A}}+\left\|\boldsymbol{\Pi}_{\boldsymbol{A}}\right\|\right) \mathrm{e}^{s(\boldsymbol{A}) t}
$$

for all $t \in \mathbb{R}_{+}$. Finally,

$$
\begin{aligned}
\left\|\frac{1}{t} \int_{0}^{t} \mathrm{e}^{-s(\boldsymbol{A}) u} \mathrm{e}^{u \boldsymbol{A}} \mathrm{d} u-\boldsymbol{\Pi}_{\boldsymbol{A}}\right\| & =\left\|\frac{1}{t} \int_{0}^{t}\left(\mathrm{e}^{-s(\boldsymbol{A}) u} \mathrm{e}^{u \boldsymbol{A}}-\boldsymbol{\Pi}_{\boldsymbol{A}}\right) \mathrm{d} u\right\| \leqslant \frac{1}{t} \int_{0}^{t}\left\|\mathrm{e}^{-s(\boldsymbol{A}) u} \mathrm{e}^{u \boldsymbol{A}}-\boldsymbol{\Pi}_{\boldsymbol{A}}\right\| \mathrm{d} u \\
& \leqslant \frac{1}{t} \int_{0}^{t} c_{\boldsymbol{A}} \mathrm{e}^{-\kappa_{\boldsymbol{A}} u} \mathrm{~d} u=\frac{c_{\boldsymbol{A}}}{\kappa_{\boldsymbol{A}} t}\left(1-\mathrm{e}^{-\kappa_{\boldsymbol{A}} t}\right) \leqslant \frac{c_{\boldsymbol{A}}}{\kappa_{\boldsymbol{A}} t} \rightarrow 0
\end{aligned}
$$

as $t \rightarrow \infty$, which implies (v).

A.4 Remark. The Internet Seminar [1] contains some of the statements of Lemma A.3.

\section{B On moments of multi-type CBI processes}

B.1 Proposition. Let $\left(\boldsymbol{X}_{t}\right)_{t \in \mathbb{R}_{+}}$be a multi-type CBI process with parameters $(d, \boldsymbol{c}, \boldsymbol{\beta}, \boldsymbol{B}, \nu, \boldsymbol{\mu})$ such that $\mathbb{E}\left(\left\|\boldsymbol{X}_{0}\right\|\right)<\infty$ and the moment condition (2.3) holds. Suppose that $\left(\boldsymbol{X}_{t}\right)_{t \in \mathbb{R}_{+}}$is irreducible. Then the following assertions hold: 
(i) if $s(\widetilde{\boldsymbol{B}})<0$, then $\lim _{t \rightarrow \infty} \mathbb{E}\left(\boldsymbol{X}_{t}\right)=-\widetilde{\boldsymbol{B}}^{-1} \widetilde{\boldsymbol{\beta}}$;

(ii) if $s(\widetilde{\boldsymbol{B}})=0$, then $\lim _{t \rightarrow \infty} t^{-1} \mathbb{E}\left(\boldsymbol{X}_{t}\right)=\boldsymbol{\Pi} \widetilde{\boldsymbol{\beta}}$;

(iii) if $s(\widetilde{\boldsymbol{B}})>0$, then $\lim _{t \rightarrow \infty} \mathrm{e}^{-s(\widetilde{\boldsymbol{B}}) t} \mathbb{E}\left(\boldsymbol{X}_{t}\right)=\boldsymbol{\Pi} \mathbb{E}\left(\boldsymbol{X}_{0}\right)+\frac{1}{s(\widetilde{\boldsymbol{B}})} \boldsymbol{\Pi} \widetilde{\boldsymbol{\beta}}$,

where $\boldsymbol{\Pi}:=\boldsymbol{\Pi}_{\widetilde{\boldsymbol{B}}} \in \mathbb{R}_{++}^{d \times d}$ is defined in (iii) of Lemma A.3.

Proof. If $s(\widetilde{\boldsymbol{B}})<0$, then, by $(\underline{2.4})$,

$$
\lim _{t \rightarrow \infty} \mathbb{E}\left(\boldsymbol{X}_{t}\right)=\left(\int_{0}^{\infty} \mathrm{e}^{u \widetilde{\boldsymbol{B}}} \mathrm{d} u\right) \widetilde{\boldsymbol{\beta}}
$$

since the decomposition

$$
\mathrm{e}^{t \widetilde{\boldsymbol{B}}}=\mathrm{e}^{s(\widetilde{\boldsymbol{B}}) t}\left(\mathrm{e}^{-s(\widetilde{\boldsymbol{B}}) t} \mathrm{e}^{t \widetilde{\boldsymbol{B}}}-\boldsymbol{\Pi}\right)+\mathrm{e}^{s(\widetilde{\boldsymbol{B}}) t} \boldsymbol{\Pi}, \quad t \in \mathbb{R}_{+},
$$

implies

$$
\left\|\mathrm{e}^{t \widetilde{\boldsymbol{B}}}\right\| \leqslant c \mathrm{e}^{-(\kappa-s(\widetilde{\boldsymbol{B}})) t}+\mathrm{e}^{s(\widetilde{\boldsymbol{B}}) t}\|\boldsymbol{\Pi}\| \rightarrow 0 \quad \text { as } t \rightarrow \infty
$$

and

$$
\begin{aligned}
\left\|\int_{t}^{\infty} \mathrm{e}^{u \widetilde{\boldsymbol{B}}} \mathrm{d} u\right\| & \leqslant c \int_{t}^{\infty} \mathrm{e}^{-(\kappa-s(\widetilde{\boldsymbol{B}})) u} \mathrm{~d} u+\left(\int_{t}^{\infty} \mathrm{e}^{s(\widetilde{\boldsymbol{B}}) u} \mathrm{~d} u\right)\|\boldsymbol{\Pi}\| \\
& \leqslant \frac{c}{\kappa-s(\widetilde{\boldsymbol{B}})} \mathrm{e}^{-(\kappa-s(\widetilde{\boldsymbol{B}}) t}+\frac{1}{-s(\widetilde{\boldsymbol{B}})} \mathrm{e}^{s(\widetilde{\boldsymbol{B}}) t}\|\boldsymbol{\Pi}\| \rightarrow 0 \quad \text { as } t \rightarrow \infty,
\end{aligned}
$$

where $c:=c_{\widetilde{\boldsymbol{B}}} \in \mathbb{R}_{++}$and $\kappa:=\kappa_{\widetilde{\boldsymbol{B}}} \in \mathbb{R}_{++}$are given in (iv) of Lemma A.3.

Further,

$$
\widetilde{\boldsymbol{B}} \int_{0}^{t} \mathrm{e}^{u \widetilde{\boldsymbol{B}}} \mathrm{d} u=\mathrm{e}^{t \widetilde{\boldsymbol{B}}}-\boldsymbol{I}_{d} \rightarrow-\boldsymbol{I}_{d} \quad \text { as } t \rightarrow \infty,
$$

hence $\int_{0}^{\infty} \mathrm{e}^{u \widetilde{\boldsymbol{B}}} \mathrm{d} u=-\widetilde{\boldsymbol{B}}^{-1}$, which yields (i).

If $s(\widetilde{\boldsymbol{B}})=0$, then, again by the decomposition (B.1), $\left\|\mathrm{e}^{u \widetilde{\boldsymbol{B}}}\right\| \leqslant c \mathrm{e}^{-\kappa t}+\|\boldsymbol{\Pi}\| \leqslant c+\|\boldsymbol{\Pi}\|$ for all $t \in \mathbb{R}_{+}$, thus (2.4) and (v) of Lemma A.3 imply (ii).

If $s(\widetilde{B})>0$, then the statement will follow from (iii) of Lemma A.3 and

$$
\lim _{t \rightarrow \infty} \mathrm{e}^{-s(\widetilde{\boldsymbol{B}}) t} \int_{0}^{t} \mathrm{e}^{u \widetilde{\boldsymbol{B}}} \mathrm{d} u=\frac{1}{s(\widetilde{\boldsymbol{B}})} \boldsymbol{\Pi} .
$$

By the decomposition (B.1),

$$
\begin{aligned}
& \left\|\mathrm{e}^{-s(\widetilde{\boldsymbol{B}}) t} \int_{0}^{t} \mathrm{e}^{u \widetilde{\boldsymbol{B}}} \mathrm{d} u-\frac{1}{s(\widetilde{\boldsymbol{B}})} \boldsymbol{\Pi}\right\| \\
& \leqslant\left\|\mathrm{e}^{-s(\widetilde{\boldsymbol{B}}) t} \int_{0}^{t} \mathrm{e}^{s(\widetilde{\boldsymbol{B}}) u}\left(\mathrm{e}^{-s(\widetilde{\boldsymbol{B}}) u} \mathrm{e}^{u \widetilde{\boldsymbol{B}}}-\boldsymbol{\Pi}\right) \mathrm{d} u\right\|+\left\|\mathrm{e}^{-s(\widetilde{\boldsymbol{B}}) t} \int_{0}^{t} \mathrm{e}^{s(\widetilde{\boldsymbol{B}}) u} \boldsymbol{\Pi} \mathrm{d} u-\frac{1}{s(\widetilde{\boldsymbol{B}})} \boldsymbol{\Pi}\right\| \\
& \leqslant c \mathrm{e}^{-s(\widetilde{\boldsymbol{B}}) t} \int_{0}^{t} \mathrm{e}^{(s(\widetilde{\boldsymbol{B}})-\kappa) u} \mathrm{~d} u+\frac{\mathrm{e}^{-s(\widetilde{\boldsymbol{B}}) t}}{s(\widetilde{\boldsymbol{B}})}\|\boldsymbol{\Pi}\| \leqslant \frac{c\left(\mathrm{e}^{-\kappa t}-\mathrm{e}^{-s(\widetilde{\boldsymbol{B}}) t}\right)}{s(\widetilde{\boldsymbol{B}})-\kappa}+\frac{\mathrm{e}^{-s(\widetilde{\boldsymbol{B}}) t}}{s(\widetilde{\boldsymbol{B}})}\|\boldsymbol{\Pi}\| \rightarrow 0
\end{aligned}
$$


as $t \rightarrow \infty$, thus we obtain (B.2), and hence, by (2.4), (iii) as well.

From Theorems 4.3 and 4.5 in Barczy et al. [5], we derive the following moment estimations for $\left(\boldsymbol{X}_{t}\right)_{t \in \mathbb{R}_{+}}$and $\left(\boldsymbol{M}_{n}\right)_{n \in \mathbb{N}}$.

B.2 Lemma. Let $\left(\boldsymbol{X}_{t}\right)_{t \in \mathbb{R}_{+}}$be a multi-type CBI process with parameters $(d, \boldsymbol{c}, \boldsymbol{\beta}, \boldsymbol{B}, \nu, \boldsymbol{\mu})$ such that $\mathbb{E}\left(\left\|\boldsymbol{X}_{0}\right\|^{q}\right)<\infty$ and

$$
\int_{U_{d}}\|\boldsymbol{z}\|^{q} \mathbb{1}_{\{\|\boldsymbol{z}\| \geqslant 1\}} \nu(\mathrm{d} \boldsymbol{z})<\infty, \quad \int_{U_{d}}\|\boldsymbol{z}\|^{q} \mathbb{1}_{\{\|\boldsymbol{z}\| \geqslant 1\}} \mu_{i}(\mathrm{~d} \boldsymbol{z})<\infty, \quad i \in\{1, \ldots, d\}
$$

with some $q \in \mathbb{N}$. Suppose that $\left(\boldsymbol{X}_{t}\right)_{t \in \mathbb{R}_{+}}$is irreducible and critical. Then

$$
\sup _{t \in \mathbb{R}_{+}} \frac{\mathbb{E}\left(\left\|\boldsymbol{X}_{t}\right\|^{q}\right)}{(1+t)^{q}}<\infty
$$

In particular, $\mathbb{E}\left(\left\|\boldsymbol{X}_{t}\right\|^{q}\right)=\mathrm{O}\left(t^{q}\right)$ as $t \rightarrow \infty$ in the sense that $\limsup _{t \rightarrow \infty} t^{-q} \mathbb{E}\left(\left\|\boldsymbol{X}_{t}\right\|^{q}\right)<\infty$.

Proof. By Theorem 4.3 in Barczy et al. [5], we have $\mathbb{E}\left(\left\|\boldsymbol{X}_{t}\right\|^{q}\right)<\infty, t \in \mathbb{R}_{+}$, and

$$
\begin{aligned}
& \mathbb{E}\left(X_{t, j}^{k}\right) \leqslant \widetilde{c}(t)^{k} \mathbb{E}\left(\left\|\boldsymbol{X}_{0}\right\|^{k}\right)+k\|\widetilde{\boldsymbol{\beta}}\| \widetilde{c}(t)^{k} \int_{0}^{t} \mathbb{E}\left(\left\|\boldsymbol{X}_{s}\right\|^{k-1}\right) \mathrm{d} s \\
&+k(k-1) \widetilde{c}(t)^{k} \sum_{i=1}^{d} c_{i} \int_{0}^{t} \mathbb{E}\left(\left\|\boldsymbol{X}_{s}\right\|^{k-1}\right) \mathrm{d} s \\
&+\widetilde{c}(t)^{k} \sum_{\ell=0}^{k-2}\left(\begin{array}{c}
k \\
\ell
\end{array}\right) {\left[\sum_{i=1}^{d} \int_{0}^{t} \mathbb{E}\left(\left\|\boldsymbol{X}_{s}\right\|^{\ell+1}\right) \mathrm{d} s \int_{U_{d}}\|\boldsymbol{z}\|^{k-\ell} \mu_{i}(\mathrm{~d} \boldsymbol{z})\right.} \\
&\left.+\int_{0}^{t} \mathbb{E}\left(\left\|\boldsymbol{X}_{s}\right\|^{\ell}\right) \mathrm{d} s \int_{U_{d}}\|\boldsymbol{z}\|^{k-\ell} \nu(\mathrm{d} \boldsymbol{z})\right]
\end{aligned}
$$

for all $k \in\{1, \ldots, q\}, \quad t \in \mathbb{R}_{+}$, and $j \in\{1, \ldots, d\}$, where $\boldsymbol{X}_{t}:=\left(X_{t, j}\right)_{j \in\{1, \ldots, d\}}$, and $\widetilde{c}(t):=\sup _{u \in[0, t]}\left\|\mathrm{e}^{u \widetilde{\boldsymbol{B}}}\right\| \leqslant c+\|\boldsymbol{\Pi}\|$ for all $t \in \mathbb{R}_{+}$due to (6.12), since $\left(\boldsymbol{X}_{t}\right)_{t \in \mathbb{R}_{+}}$is irreducible and critical. We will show (B.4) by induction with respect to $k \in\{1, \ldots, q\}$. If $k=1$, then the above estimate implies

$$
\mathbb{E}\left(X_{t, j}\right) \leqslant \widetilde{c}(t) \mathbb{E}\left(\left\|\boldsymbol{X}_{0}\right\|\right)+\|\widetilde{\boldsymbol{\beta}}\| \widetilde{c}(t) t \leqslant(c+\|\boldsymbol{\Pi}\|)\left(\mathbb{E}\left(\left\|\boldsymbol{X}_{0}\right\|\right)+\|\widetilde{\boldsymbol{\beta}}\| t\right), \quad t \in \mathbb{R}_{+} .
$$

This yields $\sup _{t \in \mathbb{R}_{+}}(1+t)^{-1} \mathbb{E}\left(\left\|\boldsymbol{X}_{t}\right\|\right)<\infty$. Further, for all $k \in\{2, \ldots, q\}, q \geqslant 2$, and $\ell \in\{0,1, \ldots, k-2\}$, we have

$$
\begin{aligned}
\int_{U_{d}}\|\boldsymbol{z}\|^{k-\ell} \nu(\mathrm{d} \boldsymbol{z}) & =\int_{U_{d}}\|\boldsymbol{z}\|^{k-\ell} \mathbb{1}_{\{\|z\|<1\}} \nu(\mathrm{d} \boldsymbol{z})+\int_{U_{d}}\|\boldsymbol{z}\|^{k-\ell} \mathbb{1}_{\{\|z\| \geqslant 1\}} \nu(\mathrm{d} \boldsymbol{z}) \\
& \leqslant \int_{U_{d}}\|\boldsymbol{z}\|^{2} \mathbb{1}_{\{\|z\|<1\}} \nu(\mathrm{d} \boldsymbol{z})+\int_{U_{d}}\|\boldsymbol{z}\|^{k} \mathbb{1}_{\{\|z\| \geqslant 1\}} \nu(\mathrm{d} \boldsymbol{z})<\infty
\end{aligned}
$$

by (4.7) (for this we use $q \geqslant 2$ ) and (B.3). The finiteness of $\int_{U_{d}}\|\boldsymbol{z}\|^{k-\ell} \mu_{i}(\mathrm{~d} \boldsymbol{z}), i \in\{1, \ldots, d\}$, follows in the same way for all $k \in\{2, \ldots, q\}, q \geqslant 2$, and $\ell \in\{0,1, \ldots, k-2\}$. Hence, by the power means inequality, the above estimate yields $\sup _{t \in \mathbb{R}_{+}}(1+t)^{-k} \mathbb{E}\left(\left\|\boldsymbol{X}_{t}\right\|^{k}\right)<\infty$. 
B.3 Lemma. Let $\left(\boldsymbol{X}_{t}\right)_{t \in \mathbb{R}_{+}}$be a multi-type CBI process with parameters $(d, \boldsymbol{c}, \boldsymbol{\beta}, \boldsymbol{B}, \nu, \boldsymbol{\mu})$ such that $\mathbb{E}\left(\left\|\boldsymbol{X}_{0}\right\|^{2 q}\right)<\infty$ and the moment conditions (‥3) hold with some $q \in \mathbb{N}$. Suppose that $\left(\boldsymbol{X}_{t}\right)_{t \in \mathbb{R}_{+}}$is irreducible and critical. Then, for the martingale differences $\boldsymbol{M}_{n}=\boldsymbol{X}_{n}-\mathbb{E}\left(\boldsymbol{X}_{n} \mid \boldsymbol{X}_{n-1}\right), \quad n \in \mathbb{N}$, we have $\mathbb{E}\left(\left\|\boldsymbol{M}_{n}\right\|^{2 q}\right)=\mathrm{O}\left(n^{q}\right)$ as $n \rightarrow \infty$, i.e., $\sup _{n \in \mathbb{N}} n^{-q} \mathbb{E}\left(\left\|M_{n}\right\|^{2 q}\right)<\infty$.

Proof. Applying Theorem 4.5 in Barczy et al. [5], we obtain

$$
\begin{aligned}
\mathbb{E}\left(M_{n, j}^{2 q} \mid \boldsymbol{X}_{n-1}=\boldsymbol{x}\right) & =\mathbb{E}\left[\left(X_{n, j}-\mathbb{E}\left(X_{n, j} \mid \boldsymbol{X}_{n-1}\right)\right)^{2 q} \mid \boldsymbol{X}_{n-1}=\boldsymbol{x}\right] \\
& =\mathbb{E}\left[\left(X_{1, j}-\mathbb{E}\left(X_{1, j} \mid \boldsymbol{X}_{0}\right)\right)^{2 q} \mid \boldsymbol{X}_{0}=\boldsymbol{x}\right]=P_{1,2 q, j}(\boldsymbol{x})
\end{aligned}
$$

for all $n \in \mathbb{N}, \quad \boldsymbol{x} \in \mathbb{R}_{+}^{d}$, and $j \in\{1, \ldots, d\}$, where $\boldsymbol{M}_{n}:=\left(M_{n, j}\right)_{j \in\{1, \ldots, d\}}$, and hence

$$
\mathbb{E}\left(M_{n, j}^{2 q} \mid \boldsymbol{X}_{n-1}\right)=P_{1,2 q, j}\left(\boldsymbol{X}_{n-1}\right),
$$

where $P_{1,2 q, j}: \mathbb{R}^{d} \rightarrow \mathbb{R}$ is a polynomial having degree at most $q$. Using Lemma B.2, this yields

$$
\mathbb{E}\left(M_{n, j}^{2 q}\right)=\mathbb{E}\left[P_{1,2 q, j}\left(\boldsymbol{X}_{n-1}\right)\right]=\mathrm{O}\left(n^{q}\right)
$$

for all $j \in\{1, \ldots, d\}$. By the power means inequality,

$$
\left(\frac{1}{d} \sum_{k=1}^{d} a_{k}^{2}\right)^{\frac{1}{2}} \leqslant\left(\frac{1}{d} \sum_{k=1}^{d} a_{k}^{2 q}\right)^{\frac{1}{2 q}}, \quad a_{1}, \ldots, a_{d} \in \mathbb{R}
$$

and hence $\left(a_{1}^{2}+\cdots+a_{d}^{2}\right)^{q} \leqslant d^{q-1}\left(a_{1}^{2 q}+\cdots+a_{d}^{2 q}\right), a_{1}, \ldots, a_{d} \in \mathbb{R}$. Then we conclude

$$
\mathbb{E}\left(\left\|M_{n}\right\|^{2 q}\right)=\mathbb{E}\left(\left(\sum_{k=1}^{d} M_{n, j}^{2}\right)^{q}\right) \leqslant d^{q-1} \sum_{k=1}^{d} \mathbb{E}\left(M_{n, j}^{2 q}\right)=\mathrm{O}\left(n^{q}\right) .
$$

\section{Convergence of random step processes}

Next we recall a result about convergence of random step processes towards a diffusion process, see Ispány and Pap [16, Corollary 2.2].

C.1 Theorem. Let $\gamma: \mathbb{R}_{+} \times \mathbb{R}^{d} \rightarrow \mathbb{R}^{d \times r}$ be a continuous function. Assume that uniqueness in the sense of probability law holds for the $S D E$

$$
\mathrm{d} \mathcal{U}_{t}=\gamma\left(t, \mathcal{U}_{t}\right) \mathrm{d} \mathcal{W}_{t}, \quad t \in \mathbb{R}_{+}
$$

with initial value $\boldsymbol{\mathcal { U }}_{0}=\boldsymbol{u}_{0}$ for all $\boldsymbol{u}_{0} \in \mathbb{R}^{d}$, where $\left(\boldsymbol{W}_{t}\right)_{t \in \mathbb{R}_{+}}$is an r-dimensional Brownian motion. Let $\left(\boldsymbol{U}_{t}\right)_{t \in \mathbb{R}_{+}}$be a solution of the $S D E$ (C.1) with initial value $\boldsymbol{U}_{0}=\mathbf{0}$. 
For each $n \in \mathbb{N}$, let $\left(\boldsymbol{U}_{k}^{(n)}\right)_{k \in \mathbb{Z}_{+}}$be a sequence of d-dimensional martingale differences with respect to a filtration $\left(\mathcal{F}_{k}^{(n)}\right)_{k \in \mathbb{Z}_{+}}$(i.e., $\left.\mathbb{E}\left(\boldsymbol{U}_{k}^{(n)} \mid \mathcal{F}_{k-1}^{(n)}\right)=\boldsymbol{U}_{k-1}^{(n)}, \quad k \in \mathbb{N}\right)$. Let

$$
\mathcal{U}_{t}^{(n)}:=\sum_{k=0}^{\lfloor n t\rfloor} \boldsymbol{U}_{k}^{(n)}, \quad t \in \mathbb{R}_{+}, \quad n \in \mathbb{N} .
$$

Suppose $\mathbb{E}\left(\left\|\boldsymbol{U}_{k}^{(n)}\right\|^{2}\right)<\infty$ for all $n, k \in \mathbb{N}$, and $\boldsymbol{U}_{0}^{(n)} \stackrel{\mathcal{D}}{\longrightarrow} \mathbf{0}$ as $n \rightarrow \infty$. Suppose that for each $T>0$,

(i) $\sup _{t \in[0, T]}\left\|\sum_{k=1}^{\lfloor n t\rfloor} \operatorname{Var}\left(\boldsymbol{U}_{k}^{(n)} \mid \mathcal{F}_{k-1}^{(n)}\right)-\int_{0}^{t} \boldsymbol{\gamma}\left(s, \mathcal{U}_{s}^{(n)}\right) \boldsymbol{\gamma}\left(s, \mathcal{U}_{s}^{(n)}\right)^{\top} \mathrm{d} s\right\| \stackrel{\mathbb{P}}{\longrightarrow} 0$

(ii) $\sum_{k=1}^{\lfloor n T\rfloor} \mathbb{E}\left(\left\|\boldsymbol{U}_{k}^{(n)}\right\|^{2} \mathbb{1}_{\left\{\left\|\boldsymbol{U}_{k}^{(n)}\right\|>\theta\right\}} \mid \mathcal{F}_{k-1}^{(n)}\right) \stackrel{\mathbb{P}}{\longrightarrow} 0$ for all $\theta>0$

where $\stackrel{\mathbb{P}}{\longrightarrow}$ denotes convergence in probability. Then $\boldsymbol{U}^{(n)} \stackrel{\mathcal{D}}{\longrightarrow} \mathcal{U}$ as $n \rightarrow \infty$.

\section{A version of the continuous mapping theorem}

For functions $f$ and $f_{n}, n \in \mathbb{N}$, in $\mathbb{D}\left(\mathbb{R}_{+}, \mathbb{R}^{d}\right)$, we write $f_{n} \stackrel{\text { lu }}{\longrightarrow} f$ if $\left(f_{n}\right)_{n \in \mathbb{N}}$ converges to $f$ locally uniformly, i.e., if $\sup _{t \in[0, T]}\left\|f_{n}(t)-f(t)\right\| \rightarrow 0$ as $n \rightarrow \infty$ for all $T \in \mathbb{R}_{++}$. For measurable mappings $\Phi: \mathbb{D}\left(\mathbb{R}_{+}, \mathbb{R}^{d}\right) \rightarrow \mathbb{D}\left(\mathbb{R}_{+}, \mathbb{R}^{q}\right)$ and $\Phi_{n}: \mathbb{D}\left(\mathbb{R}_{+}, \mathbb{R}^{d}\right) \rightarrow \mathbb{D}\left(\mathbb{R}_{+}, \mathbb{R}^{q}\right), n \in \mathbb{N}$, we will denote by $C_{\Phi,\left(\Phi_{n}\right)_{n \in \mathbb{N}}}$ the set of all functions $f \in \mathbb{C}\left(\mathbb{R}_{+}, \mathbb{R}^{d}\right)$ for which $\Phi_{n}\left(f_{n}\right) \rightarrow \Phi(f)$ whenever $f_{n} \stackrel{\text { lu }}{\longrightarrow} f$ with $f_{n} \in \mathbb{D}\left(\mathbb{R}_{+}, \mathbb{R}^{d}\right), \quad n \in \mathbb{N}$.

D.1 Lemma. Let $\left(\mathcal{U}_{t}\right)_{t \in \mathbb{R}_{+}}$and $\left(\mathcal{U}_{t}^{(n)}\right)_{t \in \mathbb{R}_{+}}, \quad n \in \mathbb{N}$, be $\mathbb{R}^{d}$-valued stochastic processes with càdlàg paths such that $\mathcal{U}^{(n)} \stackrel{\mathcal{D}}{\longrightarrow} \mathcal{U}$ as $n \rightarrow \infty$. Let $\Phi: \mathbb{D}\left(\mathbb{R}_{+}, \mathbb{R}^{d}\right) \rightarrow \mathbb{D}^{\left(\mathbb{R}_{+}, \mathbb{R}^{q}\right)}$ and $\Phi_{n}: \mathbb{D}\left(\mathbb{R}_{+}, \mathbb{R}^{d}\right) \rightarrow \mathbb{D}\left(\mathbb{R}_{+}, \mathbb{R}^{q}\right), \quad n \in \mathbb{N}$, be measurable mappings such that there exists $C \subset C_{\Phi,\left(\Phi_{n}\right)_{n \in \mathbb{N}}}$ with $C \in \mathcal{D}_{\infty}\left(\mathbb{R}_{+}, \mathbb{R}^{d}\right)$ and $\mathbb{P}(\mathcal{U} \in C)=1$. Then $\Phi_{n}\left(\mathcal{U}^{(n)}\right) \stackrel{\mathcal{D}}{\longrightarrow} \Phi(\mathcal{U})$ as $n \rightarrow \infty$.

Lemma D.1 can be considered as a consequence of Theorem 3.27 in Kallenberg [21], and we note that a proof of this lemma can also be found in Ispány and Pap [16, Lemma 3.1].

\section{References}

[1] 17th Internet Seminar 2013/2014: Positive Operator Semigroups and Applications. Organized by Bátkai, A., Fijavz, M. K. and Rhandi, A.

http://isem17.unisa.it/w/index.php/17th_Internet_Seminar 
[2] Barczy, M., Döring, L., Li, Z. and PAP, G. (2013). On parameter estimation for critical affine processes. Electronic Journal of Statistics 7 647-696.

[3] Barczy, M., Ispány, M. and PAP, G. (2011). Asymptotic behavior of unstable INAR $(p)$ processes. Stochastic Processes and their Applications 121(3) 583-608.

[4] Barczy, M., Li, Z. and PAP, G. (2015). Stochastic differential equation with jumps for multi-type continuous state and continuous time branching processes with immigration. ALEA. Latin American Journal of Probability and Mathematical Statistics 12(1) 129-169.

[5] Barczy, M., Li, Z. and PAP, G. (2015). Moment formulas for multi-type continuous state and continuous time branching processes with immigration. To appear in Journal of Theoretical Probability DOI: 10.1007/s10959-015-0605-0

[6] BARCzy, M. and PAP, G. (2014). On convergence properties of infinitesimal generators of scaled multi-type CBI processes. Available on ArXiv: http://arxiv .org/abs/1404.3658

[7] Berman, A. and Plemmons, A. J. (1994). Non-negative matrices in the mathematical sciences. SIAM, Society for Industrial and Applied Mathematics, Philadelphia.

[8] Chung, K. L. (1960). Markov chains with stationary transition probabilities. SpringerVerlag, Berlin-Göttingen-Heidelberg.

[9] Duffie, D., Filipović, D. and Schachermayer, W. (2003). Affine processes and applications in finance. The Annals of Applied Probability 13 984-1053.

[10] Dunford, N. and Schwartz, J. T. (1958). Linear Operators. I. General theory. Interscience Publishers, New York and London.

[11] Ethier, S. N. and Kurtz, T. G. (1986). Markov processes. Characterization and convergence. Wiley, New York.

[12] Horn, R. A. and Johnson, Ch. R. (2013). Matrix Analysis, 2nd ed. Cambridge University Press, Cambridge.

[13] Huang, J., Ma, C. and Zhu, C. (2011). Estimation for discretely observed continuous state branching processes with immigration. Statistics and Probability Letters 81 11041111.

[14] Ikeda, N. and Watanabe, S. (1989). Stochastic Differential Equations and Diffusion Processes, 2nd ed. North-Holland/Kodansha, Amsterdam/Tokyo.

[15] IspÁny, M. and PAP, G. (2007). Weak convergence of step processes and an application for critical multi-type branching processes with immigration. Available on the arXiv: http://arxiv.org/abs/math/0701803v1

[16] Ispány, M. and PAP, G. (2010). A note on weak convergence of random step processes. Acta Mathematica Hungarica 126(4) 381-395. 
[17] IspÁny, M. and PAP, G. (2014). Asymptotic behavior of critical primitive multi-type branching processes with immigration. Stochastic Analysis and Applications 32(5) 727741.

[18] Iyer, G., Leger, N. and Pego, R. L. (2015). Limit theorems for Smoluchowski dynamics associated with critical continuous-state branching processes. The Annals of Applied Probability 25(2) 675-713.

[19] Jacod, J. and Shiryaev, A. N. (2003). Limit Theorems for Stochastic Processes, 2nd ed. Springer-Verlag, Berlin.

[20] Jagers, P. (1969). The proportions of individuals of different kinds in two-type populations. A branching process problem arising in biology. Journal of Applied Probability 6(2) 249-260.

[21] Kallenberg, O. (1997). Foundations of Modern Probability. Springer, New York, Berlin, Heidelberg.

[22] Karatzas, I. and Shreve, S. E. (1991). Brownian Motion and Stochastic Calculus, 2nd ed. Springer, Berlin.

[23] Li, Z. (2011). Measure-Valued Branching Markov Processes. Springer-Verlag, Heidelberg.

[24] Mullikin, T. W. (1963). Limiting distributions for critical multitype branching processes with discrete time. Transactions of the American Mathematical Society 106 469-494.

[25] Ren, Y.-X., Yang, T. and Zhao, G.-H. (2014). Conditional limit theorems for critical continuous-state branching processes. Science China Mathematics 57(12) 2577-2588.

[26] Ren, Y.-X., Song, R. and Zhang, R. (2014). Limit theorems for some critical superprocesses. Available on ArXiv: http://arxiv.org/abs/1403.1342

[27] Revuz, D. and Yor, M. (2001). Continuous martingales and Brownian motion, 3rd edition, corrected 2nd printing. Springer-Verlag, Berlin.

[28] Wei, C. Z. and Winnicki, J. (1989). Some asymptotic results for the branching process with immigration. Stochastic Processes and their Applications 31(2) 261-282.

[29] Yakovlev, A. Y. and Yanev, N. M. (2010). Limiting distributions for multitype branching processes. Stochastic Analysis and Applications 28(6) 1040-1060. 